\title{
V. Die Sowjetunion und das Problem der Sicherheit: Die Planungen im „Post-Hostilities Planning Staff“ und im Foreign Office
}

\section{Die Allianz mit der Sowjetunion als Strukturproblem der britischen Deutschlandplanungen}

Es wurde bereits darauf hingewiesen, daß im Unterschied zu den Planungen bis zur Bildung des Economic and Industrial Planning Staff, die durch den Entwurf keynesianisch geprägter Blaupausen für die wirtschaftliche Behandlung Deutschlands nach dem Krieg gekennzeichnet und überwiegend auf den Kreis der Experten begrenzt geblieben waren, mit der Reorganisation der Planungsarbeit, der damit verbundenen Verlagerung auf die Ebene des Kabinetts und seiner Ausschüsse sowie dem sich abzeichnenden Sieg der Alliierten im Frühjahr 1944 in wachsendem Maße politische und militärische Erwägungen in den Planungsprozeß eindrangen. Sichtbarer Ausdruck hierfür waren OCTAGON und die Auseinandersetzung um den Morgenthauplan gewesen. Mit dem Vordringen der alliierten Truppen und der Roten Armee rückte schließlich mehr und mehr die Frage nach dem Verhältnis der Westmächte zur Sowjetunion und der daraus zu ziehenden Konsequenzen für die Formulierung der Deutschlandpolitik in den Mittelpunkt der Überlegungen.

Die britischen Nachkriegsplanungen gegenüber Deutschland, so wie sie sich bis zur Potsdamer Konferenz herauskristallisiert hatten, basierten unausgesprochen auf der Annahme, daß die Zusammenarbeit mit Moskau auch beim Aufbau und der sicherheitspolitischen Untermauerung der Friedensordnung fortdauern würde. Gerade der bislang erreichte Planungskonsens, von der Teilung und Zerstückelung Deutschlands abzusehen und im Hinblick auf einen reibungslosen Ablauf der Reparationsentnahmen sowie zur Sicherstellung der Ernährungslage die wirtschaftliche Einheit aufrechtzuerhalten, hing aber von der weiteren Kooperationsbereitschaft des Kreml ab.

In diesem Zusammenhang ist immer wieder ein Vorgang des Sommers 1944 in den Vordergrund des historischen Interesses gerückt. Stein des Anstoßes waren Überlegungen der britischen Stabschefs im Rahmen ihrer langfristigen militärstrategischen Planungen, die die Sowjetunion als zukünftige Hauptbedrohung herausstellten. Die Tatsache, daß es das Foreign Office im Verlauf der Auseinandersetzung nicht mehr zu einer Überbrückung der Gegensätze mit den Stabschefs kommen ließ, war ein eindrucksvoller Beleg für die Dominanz des Außenministeriums im Verhältnis zu den Militärs. Allerdings lag das primäre Interesse der Historiker bei der Rekonstruktion dieses Konflikts bisher darauf, seine Bedeutung für die Diskussion innerhalb der Verteidigungsorganisation Londons ${ }^{1}$, für die Entwicklung der britisch-sowjetischen Beziehungen ${ }^{2}$ oder die Frage der Teilung Deutschlands ${ }^{3}$ herauszuarbeiten. Cairncross, der die Reparationsproblematik in den Mittelpunkt seiner Darstellung stellt, wertet die Auseinandersetzung zwi-

\footnotetext{
1 Lewis, S. 98-177.

${ }^{2}$ Ross, S. 521-540; Rothwell, S. 114-123.

${ }^{3}$ Kettenacker, Krieg zur Friedenssicherung, S. 479-486; Tyrell, S. 444-458.
} 
schen Chiefs of Staff und Foreign Office als weiteres Indiz dafür, daß es Whitehall nicht gelang, bis zur Konferenz von Jalta eine Einigung in der Dismemberment- und Reparationsfrage zu erzielen ${ }^{4}$. Demgegenüber ist der enge Bezug dieses Konflikts zu dem Problem der „Economic Security“ nicht gebührend herausgestellt worden. Wichtige Hinweise hierfür liefert Ebersold, der den Konflikt in den Kontext der allgemeinen sicherheitspolitischen Orientierungssuche stellt und ihn als konzeptionellen Grundlagenstreit zwischen Vertretern der Idee eines kollektiven Sicherheitssystems und den Verteidigern eines regionalen, in Konfrontation zur Sowjetunion stehenden Bündnisses wertet ${ }^{5}$.

Betrachtet man die anglo-sowjetischen Beziehungen während des Zweiten Weltkriegs und insbesondere die Einschätzung der Politik und Intentionen des Kreml durch die Beamten des Außenministeriums genauer, so fällt die mit Schärfe und Entschiedenheit vorgetragene Reaktion auf, mit der jegliche Planspiele über eine mögliche Frontstellung gegen Moskau zurückgewiesen wurden; diesem Befund kommt um so größere Beachtung zu, als das Verhalten des Foreign Office in diesem Punkt in auffallendem Gegensatz zur Zurückhaltung bei anderen Grundsatzentscheidungen steht. Dabei hatte zu Beginn des Krieges noch großes Mißtrauen über die Intentionen der UdSSR bestanden. Die britischen Beziehungen Londons zu Moskau waren nicht nur durch die Ereignisse des Sommers 1939 schwer belastet, die das rücksichtslose Finassieren Stalins für die britischen Politiker und Diplomaten offenbart hatten; das angeknackste Verhältnis mochte zwar durch das gemeinsame Ziel der militärischen Niederwerfung Hitlers temporär verdeckt werden, konnte jedoch schwerlich eine tragfähige Basis für die dauerhafte Zusammenarbeit nach Kriegsende bilden. Vielmehr sorgten Stalin und sein Außenminister Molotow auch während des Krieges immer wieder dafür, daß Skepsis und Argwohn in Whitehall nicht verstummten. So ließen sie kaum eine Gelegenheit aus, Fragen der territorialen Neuordnung Osteuropas anzuschneiden und ihre britischen Gesprächspartner zur Anerkennung der Annexion der baltischen Staaten zu drängen. Auch bei seiner MoskauReise im Dezember 1941 wurde Eden von dem Vorschlag Stalins überrascht, im Rahmen eines gemeinsamen Abkommens, in dem sich beide Staaten zu gegenseitiger militärischer Unterstützung sowie zur Zusammenarbeit beim Wiederaufbau verpflichten sollten, das Grenzthema ganz in der Manier des Hitler-Stalin-Pakts zum Gegenstand eines geheimen Protokolls zu machen'.

Es gab denn auch unter den Beamten angesichts der eigenen schwierigen militärischen Lage, die einen Konfrontationskurs mit der Sowjetunion kaum ratsam erscheinen ließ, und den anfänglichen Erfolgen des Unternehmens Barbarossa, die zumindest in der ersten Kriegshälfte die Gefahr einer deutschen Totalhegemonie Europas wahrscheinlicher machten als die einer russischen Teilhegemonie über Osteuropa, zunächst kaum Dissens darüber, daß Moskau nach Kriegsende umfangreiche, auch territorial definierte Sicherheitsansprüche geltend machen würde 7 . Soweit diese Zielvorstellung im Außenministerium und im Kabinett akzeptiert war, wurde sie begleitet von der Frage, ob es gelingen konnte, ohne amerikanischen Beistand die völlige Sowjetisierung dieser Länder zu ver-

${ }_{4}^{4}$ Cairncross, The Price of War, S. 44-49.

${ }^{5}$ Ebersold, S. 95-97.

6 Vgl. Rothwell, S. 76-78, 85-90, 95; Carlton, S. $191 \mathrm{f}$.

$7 \mathrm{Zu}$ den Territorialverschiebungen auf der Basis der Grenzen von 1941 in diesem Zusammenhang vgl. Foschepoth, Großbritannien, S. 65-85; Kettenacker, Krieg zur Friedenssicherung, S. 105112; Blasius, S. XIII-XXII. 
hindern und gleichzeitig die eigene Einflußnahme auf den Wiederaufbau Osteuropas zu wahren. Einen ersten Schritt hierzu hofften die Foreign Office-Beamten mit dem Abschluß des britisch-sowjetischen Vertrages vom 26. Mai 1942 getan zu haben, zumal Molotow zur Verblüffung Londons auf eine Erwähnung der Grenzproblematik verzichtete. Statt eines Grenzvertrags vereinbarte man ein bilaterales Bündnis, das ein Wiederaufleben deutscher Aggression nach dem Krieg verhindern sollte ${ }^{8}$. Im Außenministerium gab man sich aber bereits zu einem frühen Zeitpunkt keinen Illusionen mehr über die Alternative einer Kooperation mit Moskau hin: Sollte, so wurde gefolgert, die Sowjetunion aus dem Krieg ebenfalls als Hegemonialmacht hervorgehen und durch eine rücksichtslose Einflußsphärenpolitik in Osteuropa den Konflikt mit dem Westen heraufbeschwören, so sähe sich Großbritannien außerstande, dem etwas entgegenzusetzen. Vielmehr zogen die Foreign Office-Planer den für sie deprimierenden Schluß, daß für diesen Fall die erneute Mobilisierung Deutschlands unausweichlich sein würde; dies war um so wichtiger, als ein Ausgreifen Moskaus auf die wirtschaftlichen Ressourcen Deutschlands und damit ein zweites Rapallo verhindert werden mußte9.

Der Vertrag mit Moskau ${ }^{10}$ war auf den ersten Blick eine logische Konsequenz aus dem Kriegsverlauf, der die im Januar 1943 auf der Konferenz von Casablanca mit den USA vereinbarte „Germany first “-Strategie ${ }^{11}$ entsprach. Ungeachtet eines „die Weltpolitik im Verlauf des 19. und 20. Jahrhunderts in entscheidendem Maße“ prägenden „machtpolitischen Antagonismus zwischen England und Rußland bzw. der Sowjetunion“12 ließen die Briten keinen Zweifel daran, daß der Kriegsgegner Deutschland hieß. Die im Verlaufe des Krieges immer populärere Allianz mit dem Kreml spiegelte schließlich auch den „fast bis zur Bewegungslosigkeit geschrumpften “ Handlungsspielraum Großbritanniens wider, über dessen Möglichkeiten sich Hitler wie die Kreise des Widerstands gleichermaßen Illusionen hinsichtlich eines deutsch-britischen Rapprochements hingaben ${ }^{13}$. Entscheidend war jedoch, daß der Vertrag hinsichtlich des ihm zugrunde liegenden Sicherheitsmodells für die Nachkriegszeit eine Fortdauer der eigentlich zweckgebundenen militärischen Allianz mit Moskau auf der Basis traditioneller Bündnisvorstellungen und eines bilateralen Interessenausgleichs als Mittel britischen Machterhalts nicht ausschloß. Seine doppelte Zielvorgabe, durch eine Allianz mit der Sowjetunion die deutsche Gefahr einzudämmen, gleichzeitig aber mit einer Einbindung Moskaus Rapallo-Ängste zu besänftigen, reflektierte gleichermaßen den weiterhin reklamierten Machtanspruch wie dessen mangelnde wirtschaftliche Tragfähigkeit, und enthielt deutliche Elemente der bekannten Appeasement-Politik.

Dies wurde besonders deutlich, als in dem von Leith-Ross geleiteten „Inter-Allied Post-War Requirements Committee " im Verlaufe einer stürmischen Sitzung der Vertreter des Außenministeriums zur Rechenschaft gezogen wurde. Von Keynes und LeithRoss angegriffen, fühlte sich Jebb zur Verteidigung des Vertragswerks herausgefordert.

\footnotetext{
${ }^{8}$ Vgl. Memorandum by the Secretary of State for Foreign Affairs, „USSR“, 12.5.1942, in: DzD I/3, S. 342-345.

${ }^{9}$ Vgl. Ross, S. 524.

${ }^{10}$ Für den Wortlaut vgl. Deuerlein, S. 304-306. Die beste Darstellung bei Blasius, S. XXII-XXXI, sowie Kettenacker, Krieg zur Friedenssicherung, S. 112-130.

11 Vgl. Henke, S. 44-66.

12 Hildebrand, Das vergangene Reich, S. 822.

${ }^{13}$ Ebenda, S. 805-813, 820-822 (Zitat S. 807).
} 
In programmatischer Weise erklärte er den Ökonomen, in den Worten Daltons, „that no-one except the F.O. officials, who sent telegrams about our relations with Russia, realised how difficult and, indeed, dangerous, these were. Therefore, it was most important to do all we could to conciliate the Russians, nor would it be a bad thing to let the Americans know that we were paying heed to the Russians and had a second string to our bow. " ${ }^{14}$ Die Entrüstung der beiden Treasury-Mitarbeiter, insbesondere von Keynes, der gegen Ronald und Jebb polemisierte, das Außenministerium solle sich einen neuen Rechtsberater zulegen, mußte auf den ersten Blick verblüffen. Was veranlaßte zwei Ökonomen zu einer so scharfen Kritik, versprach doch das Vorgehen des Außenministeriums das Verhältnis der Alliierten mit dem Ziel der militärischen Zerschlagung des Nationalsozialismus zu stabilisieren?

Das Abkommen mit der Sowjetunion, mit dem man konkret die drohende Alternative des Wiederaufbaus Deutschlands und seine sicherheitspolitische Integration als Puffer in Frontstellung zur Sowjetunion abwenden sowie eine Sicherheirsoption im Falle des isolationistischen Rückfalls der USA nach Kriegsende offenhalten wollte, war der sichtbare äußere Ausdruck des ebenfalls im Frühjahr 1942 entstandenen, im wesentlichen von Jebb formulierten „Four Power Plan"15. Die darin angestellten Überlegungen ließen die tieferen Motive erkennen, die eine britisch-sowjetische Allianz mit traditionellen Elementen der britischen Außenpolitik verknüpften. Kern des Plans war die sicherheitspolitische Befriedung Europas unter den Auspizien Londons und Moskaus mit den Mitteln der Regionalisierung bzw. Föderalisierung. Dahinter verbarg sich nichts anderes als der Wunsch nach Wiederherstellung der Bedingungen für eine klassische Einflußsphärenpolitik, die Großbritannien einen Ausweg aus dem Teufelskreis von strategischer Überdehnung und Ressourcenknappheit zu versprechen schien und den drohenden Machtverlust abwenden konnte. Gleichzeitig sollte die drohende Gefahr eines kontinentalen Engagements möglichst gering gehalten und Londons Position im Konzert der Großmächte garantiert werden: Mochte auch die Zusammenarbeit mit den USA weiterhin Priorität besitzen, angesichts der Unsicherheit über deren Politik nach Kriegsende schien es jedenfalls sinnvoll, ihnen weiterhin den westatlantischen und pazifischen Einflußbereich zu überlassen, während in Europa Großbritannien selbst als Schutzmacht des westlichen Teils des Kontinents auftreten würde; der Sowjetunion stand es frei, durch ein Bündnis mit den osteuropäischen Staaten sowie eine ins Auge gefaßte Balkanföderation den eigenen Einflußbereich zu sichern, um, in Jebbs Worten, die Formierung dieser Staaten gegen einen erneuten deutschen „Drang nach Osten“ zu organisieren.

Jebb ließ keinen Zweifel daran, daß der direkte Zweck des Plans die dauerhafte Entmachtung Deutschlands, das eigentliche Ziel freilich die Bewahrung der britischen

\footnotetext{
${ }^{14}$ BLPES, Dalton Diaries I/27, fol. 86 f. (Eintrag 7.10.1942). Jebb griff hier auf ein im Rahmen seines Viermächteplans immer wieder in FO-Kreisen geäußertes Bild zurück. So schrieb etwa Sargent im August 1942, man dürfe in Verhandlungen mit den USA nicht Moskau Anlaß zu Mißtrauen geben, „lest we make it impossible for ourselves to collaborate with her if the United States fails us. The Soviet connexion must always remain a second string to our bow, and we must be careful therefore not to mislay this string while practising our archery with the Americans." Sargent an Jebb, 23.8.1942, zitiert nach: Kettenacker, Krieg zur Friedenssicherung, S. 139.

${ }^{15}$ Für den Wortlaut vgl. Woodward, British Foreign Policy, Bd. 5, S. 1-21, sowie DzD 1/3, S. 743764.
} 
Großmachtstellung war ${ }^{16}$. Im Außenministerium hatte man richtig erkannt, daß nur die Fortsetzung der Kriegskoalition wenn schon nicht den Status Großbritanniens als Primus inter pares, so doch wenigstens die machtpolitische Gleichberechtigung des Landes an der Seite der beiden Flügelmächte garantieren konnte. Dies wiederum schloß eine derartige Gleichberechtigung Deutschlands bzw. Japans aus, und zu Recht wies Jebb auf den darin begründeten fundamentalen Unterschied zwischen einer Vier- bzw. Dreimächtekontrolle und dem kollektiven Sicherheitsmodell des Völkerbunds nach dem Ersten Weltkrieg hin. Aus dem streng machtpolitischen Blickwinkel des Außenministeriums lautete die Gleichung, daß die Aufgabe des Feindbildes Deutschland die Einheit der Alliierten in seinen Grundfesten gefährdete und letztlich das Absinken der Inselmacht auf den Status des Juniorpartners der neuen Führungsmacht USA und damit das Eingeständnis des endgültigen imperialen Abstiegs nach sich ziehen mußte ${ }^{17}$.

Auch wenn aus der Foreign Office-Perspektive die einem britisch-russischen Ausgleich zugrunde liegenden Antriebsmomente prinzipiell erkannt waren, das Konzept richtete sich als ganzes doch gleich in doppeltem Sinn gegen die aus dem Umkreis von Keynes wiederholt vorgetragenen deutschlandpolitischen Kerngedanken. Eine auf die Erneuerung des Status quo ante zielende Großmachtpolitik war nicht in der Lage, in dem Scheitern von Versailles das Versäumnis zu erblicken, das internationale Mächtesystem nach 1919 auf der Grundlage umfassender Reformmaßnahmen neu zu ordnen. Vielmehr schien gerade das Versagen des internationalen Kontrollsystems und die darin begründete Unfähigkeit der Großmächte, über alle ideologischen Grundsätze hinweg zu einer wirksamen „Eindämmung“ der Hitlerschen Gefahr zu finden, den Weg in den Zweiten Weltkrieg geebnet zu haben. Weniger auf eine friedensvertragliche Neuordnung fixiert, die notwendigerweise auch eine Neudefinition des Mächtestatus von Großbritannien implizierte, erblickten die Beamten erneut in (gemäßigten) reparationspolitischen und territorialen Ausbesserungsarbeiten eine Möglichkeit, einen kontinentaleuropäischen Interessenausgleich auf der Basis einer völlig neuartigen Mächtekonstellation zu schaffen und damit die Vorrangstellung Großbritanniens zu garantieren ${ }^{18}$.

Daneben ging Jebb in seinem Plan von einem deutlichen Primat der imperialen Position Großbritanniens vor innenpolitischen Reformen aus. Explizit übte er Kritik an jener Denkrichtung, die von einer Selbstbescheidung der britischen Weltmachtrolle zugunsten sozio-ökonomischer Reformmaßnahmen ausging, wie sie der Beveridge-Re-

16 Aus diesem Grund stand man dem Verlangen der Amerikaner, China als vierte Garantiemacht eines internationalen Sicherheitssystems aufzunehmen, auch eher ablehnend gegenüber. Anstatt die östliche Macht in den Kreis der Rooseveltschen "Vier Polizisten“ aufzunehmen, zog man im Foreign Office fraglos eine machtpolitische Beförderung Frankreichs vor, das als kontinentale Führungsmacht unter dem Dach des Viererclubs in einer Art Neuauflage der französisch-russischen Entente von 1892/94 und der Tripleentente von 1907 mit militärischer und handelspolitischer Stoßrichtung gegen Deutschland nicht nur zu dessen Niederhaltung, sondern gleichzeitig auch mit Domestizierungswirkung auf Moskau zur Wiederherstellung des Mächte-Status quo in Europa beitragen konnte und Großbritannien die Hände frei ließ für die Fortsetzung seiner auBereuropäischen Rolle.

${ }_{17}$ Zum „Four Power Plan“ vgl. insbesondere die ebenfalls das machtpolitische Kalkül des Außenministeriums betonende Analyse bei Kettenacker, Krieg zur Friedenssicherung, S. 130-146, sowie ders., The Anglo-Soviet Alliance, S. 435-458. Vgl. ferner Woodward, British Foreign Policy, S. 434 f.; Tyrell, S. 64-83, 92-98; Lewis, S. 35-39; Ebersold, S. 84 f.

18 Vgl. vor allem die Hinweise bei Kettenacker, Krieg zur Friedenssicherung, S. 183 f.; sowie zusammenfassend ders., Die anglo-amerikanischen Planungen, S. 66-87, besonders S. $71 \mathrm{f}$. 
port umrissen hatte. Es stand außer Frage, daß hier zwei sich gegenseitig ausschließende Ansprüche miteinander konkurrierten. Genau hier stand eine Entscheidung an über die seit dem Ausgang des letzten Jahrhunderts konvergierenden Linien britischer Außenpolitik: Die Bewahrung der Reformfähigkeit und die Einlösung des Anspruchs auf Friedensbewahrung setzte nämlich gerade diese Selbstbescheidung des Landes im Rahmen des wirtschaftlich Möglichen voraus, auch um den Preis eines Bruchs mit der Sowjetunion, der Überlassung der Führungsposition an die USA und einer Stabilisierungspolitik gegenüber Deutschland. Umgekehrt bedrohte das Beharren auf einem Primat der Außenpolitik, das den Frieden im Äußeren mit den Mitteln klassischer Großmachtpolitik auf der Basis eines west-östlichen Ausgleichs zu erreichen suchte und die Kontinuität des imperialen Status Großbritanniens voraussetzte, die vielbeschworene soziale Sicherheit im Inneren. Obwohl also die Beamten des Außenministeriums mit ihrem Plan einer Viermächte-Kontrolle Deutschlands und der Beschwichtigung Moskaus konsequent in der Tradition britischer Außenpolitik zu stehen meinten, erkannten sie augenscheinlich nicht den inneren Widerspruch ihrer Konzeption, der die Dualität von Reform und Frieden als außenpolitische Grundziele aufzulösen drohte. Sie entschieden sich jedenfalls ohne zu zögern für eine deutschlandpolitische Allianz mit dem Kreml, und Jebb warf in seiner Denkschrift konsequenterweise allen, die demgegenüber einen Primat der Innenpolitik vertraten, Defätismus vor ${ }^{19}$.

Keynes' Bedenken zielten mithin auf die antideutsche Ausrichtung des britisch-sowjetischen Vertrags, die in einem fundamentalen gedanklichen Gegensatz zu dem gerade im Entstehen begriffenen „Economic Security“-Konzept stand und den Wiederaufbaugedanken dem Ziel außenpolitischen Machterhalts unterordnete. Der vom Außenministerium favorisierte Rückgriff auf Mittel der klassischen Machtpolitik konnte kaum auf die Gegenliebe des Ökonomen stoßen, dem immer stärker die schwindende britische Wirtschaftsbasis und folglich die Notwendigkeit eines Teilrückzugs Londons aus der internationalen Verantwortung ins Bewußtsein rückte. Sie liefen seinem Lösungsmodell der weltwirtschaftlichen und machtpolitischen Neuordnung sowie dem Vorrang der inneren Stabilisierung mit Mitteln budgetärer Expansion zuwider. Es stand also zu befürchten, daß sich in den politisch-strategischen Planungen des Außenministeriums ein Widerspruch zu entfalten begann zwischen der Einsicht in die Unabdingbarkeit einer Stabilisierungspolitik gegenüber Deutschland, die man aus dem Scheitern der Versailler Friedensordnung ableitete, und dem Gedanken, daß nur eine Fortschreibung der imperialen Rolle des Königsreichs und eine Bündnispolitik gegenüber Moskau einen dauerhaften Frieden garantieren könnten.

Der „Tag der Roten Armee“, der im Februar 1943 in der britischen Bevölkerung eine ungeahnte Solidaritätswelle für die sowjetischen Soldaten auslöste, verankerte die Kooperationspolitik des Außenministeriums auf breiter Basis und machte sie zu einem Anliegen nahezu des gesamten politischen Spektrums. Die von Dalton in Birmingham vorgetragene Einschätzung, bei dem Vertrag handele es sich um „the best treaty any British

${ }^{19} \mathrm{Vgl}$. die Argumentation bei Kettenacker, Krieg zur Friedenssicherung, S. 134 f., der die Stoßrichtung des Foreign Office gegen Labour betont. Wie sich bald herausstellen sollte, beging Labour freilich den umgekehrten Fehler: Hier wurde der Anspruch auf Sozialreformen mit einer harten Politik gegenüber Deutschland verbunden und dabei dessen Abhängigkeit von einer Stabilisierungspolitik genauso übersehen wie in Jebbs Viermächteplan im Zusammenhang mit dem Anspruch auf Friedenswahrung. 
Foreign Secretary has signed in our lifetime“, spiegelte den sich abzeichnenden außenpolitischen Konsens unter den Politikern wie in der Bevölkerung. Er fügte hinzu: „Historical necessity has thrown the Russians and ourselves into an comradeship which must long outlast our common victory in this war." 20

Eine Bestätigung seiner Politik fand das Foreign Office zunächst in den strategischen Planungen der Militärs. Das beharrliche Drängen insbesondere einer Reihe exilierter norwegischer, holländischer und belgischer Politiker in der Frage eines möglichen atlantischen Sicherheitsbündnisses nach dem Krieg veranlaßte das Außenministerium im Januar 1942, also genau zwischen Edens Moskau-Reise und dem Abschluß des britischsowjetischen Vertrags, die Stabschefs um ein Grundsatzpapier über die militärisch-strategische Orientierung Großbritanniens zu bitten. In ihrem Anfang April vorgelegten „Basenplan" bestätigte die militärische Führung, daß eine künftige Bedrohung Europas nur von einem erneuten Aufstieg Deutschlands ausgehen könne. Ein wirksamer Schutz bestand in ihren Augen daher lediglich in der Unterhaltung ausreichender Streitkräfte und dem erklärten Willen, sie notfalls erneut gegen Deutschland einzusetzen. Der Zustand völliger Verteidigungslosigkeit, in den die Deutschen auf unabsehbare Zeit zu versetzen waren, legte ihrer Meinung nach den Schwerpunkt des Einsatzes auf die britischen Luftstreitkräfte. Die Herausstellung eines kontinentalen Hauptfeindes in der Mitte Europas ließ die Planer denn auch ein Militärbündnis mit Norwegen skeptisch beurteilen, das aus ihrer Sicht Großbritannien strategisch zu weit an die nördliche Hemisphäre binden würde und deshalb ohne ein ergänzendes Engagement der USA gar nicht zu realisieren war. Demgegenüber beurteilten sie eine engere verteidigungspolitische Zusammenarbeit mit den kontinentalen Randstaaten im Westen als günstiger ${ }^{21}$.

Der sich an dieses Papier anknüpfende Dialog zwischen den Beamten des Außenministeriums und den Vertretern der Streitkräfte machte bei aller Sympathie, die die Beamten dieser Interpretation entgegenbringen mußten, deutlich, daß nicht unähnlich den Planungen über die wirtschaftliche Behandlung Deutschlands ein Interessenkonflikt zwischen der Experten- und Entscheidungsebene im Entstehen begriffen war. Während das Foreign Office in den Militärs primär Ansprechpartner zur Ausarbeitung von langfristigen regionalen Strategiestudien sah, insistierten deren Planer darauf, daß ohne vorherige Entscheidungen über außenpolitische Leitlinien für die Nachkriegszeit Studien allgemeineren Charakters wenig sinnvoll seien, und warnten vor Regionallösungen, die einer generellen Analyse der strategischen Folgen des Weltkriegs vorausgingen. Zur besseren Koordination der Gesamtplanungen mit den militärischen Zielvorstellungen entstand das Military Sub-Committee (MSC) ${ }^{22}$.

Das MSC wurde vom Außenministerium sofort darum gebeten, die Möglichkeiten des „Basenplans“ im Hinblick auf eine Gewinnung der USA als Schutzmacht des atlantischen Raums an der Seite Großbritanniens auszuloten. Die wenig später vorgelegte Aufzeichnung bestätigte erneut die Grundsätze des britisch-sowjetischen Abkommens. Die Planer gingen weiterhin von der Annahme aus, daß Deutschland der primäre Bedrohungsfaktor in Westeuropa sei. Sie unterstrichen die Notwendigkeit seiner dauerhaften

20 BLPES, Dalton Papers II, 7/5, fols. 23-26, hier: fol. 24, Daltons Rede in Birmingham, 20.2.1943. Noch im Dezember 1944 bezeichnete er den Vertrag als "the anchor of post-war peace in Europe“; BLPES, Dalton Diaries I/31, fol. 96 (Eintrag 12.12.1944).

${ }^{21}$ Zum Basenplan vgl. Lewis, S. 5-9.

22 Zur Entstehung des MSC vgl. ebenda, S. 17-24. 
Entwaffnung unter der Kontrolle der Besatzungsarmeen, denen zur strategischen Absicherung Nachschubbasen in Deutschland und den Niederlanden an die Seite gestellt werden sollten. Die Idee britischer Militärbasen in Skandinavien und Westeuropa wurde demgegenüber zwar unter Hinweis auf ihren Wert als zusätzliche Sicherheitsmaßnahme im Falle des Scheiterns einer dauerhaften Entmachtung Deutschlands nicht völlig verworfen, jedoch als langfristig kostspieliges kontinentales Engagement bewertet. Es sei offensichtlich, daß die zur wirkungsvollen Sicherheit dieser Länder notwendigen Kosten nur mit einer umfangreichen Eigenleistung und unter der Voraussetzung einer Beteiligung der USA zu tragen seien. Diese ersten, noch vorsichtig vorgetragenen Erwägungen aus militärstrategischer Sicht gelangten im Rahmen einer Vorlage des Außenministeriums immerhin ins Kabinett, fanden jedoch weder die Billigung der Minister noch die der Planungsdirektoren der Stabschefs, die übereinstimmend die Ausarbeitung von Strategieplänen vor einer ministeriellen Übereinkunft über die politischen Rahmenbedingungen für verfrüht hielten ${ }^{23}$.

Mochten auch in der Folgezeit keine weiteren Entscheidungen fallen, die eine rasche Wiederaufnahme der militärischen Planungen ermöglichten, so hatten die ersten Vorstöße im zeitlichen Umfeld des britisch-sowjetischen Abkommens doch zumindest den breiten außenpolitischen Konsens in Whitehall, die Kriegskoalition mit Moskau über das Kriegsende hinaus als Voraussetzung für den Fortbestand des Großmachtstatus Großbritanniens fortzusetzen, auch aus strategischer Sicht bestätigt. Weiteren Überlegungen wurde damit aus Foreign Office-Sicht vorerst jede Dringlichkeit genommen. So hielt der Leiter des mit Rußlandfragen befaßten Northern Department, Christopher Warner, im Juni 1943 in seiner Beurteilung eines weiteren MSC-Papiers fest, Großbritanniens Politik "was certainly to go all out for Russian co-operation, and the Russian response so far was encouraging "24. Warner regte an, eine Kooperationsbereitschaft der Sowjetunion als Arbeitshypothese den Nachkriegsplanungen zugrunde zu legen.

\section{Planungsgegensätze: Sicherheit auf europäischer Ebene oder Großmachtstatus?}

Die Situation blieb bis zum Frühjahr 1944 unverändert. Zuvor schien sich das Konzept des Außenministeriums für alle sichtbar zu bestätigen, als Eden auf der Moskauer Außenministerkonferenz im Oktober und Churchill in Teheran im November 1943 noch einmal Großbritanniens Führungsanspruch demonstrieren konnten und dafür selbst das Lob der nur mangelhaft vorbereiteten Amerikaner erhielten ${ }^{25}$. $\mathrm{Zu}$ dem aus britischer Sicht erfolgreichen Abschluß der Konferenzen hatte vor allem beigetragen, daß der Kreml mit seiner Zustimmung zur Schaffung einer Europäischen Beratenden Kommission mit Sitz in London und zu einer „Viermächte-Erklärung“, in der die Kriegsalliierten sich über ein gemeinsames Vorgehen in der Frage der Entwaffnung und Besetzung Deutschlands sowie über die Schaffung einer internationalen Organisation zum frühest

${ }^{23}$ Ebenda, S. 24-30.

24 Zitiert nach: ebenda, S. 42.

$25 \mathrm{Vgl}$. Hull an Eden, 30.10.1943, in: Carlton, S. 227. Vgl. auch Rothwell, S. 108 f., sowie, auch zum Folgenden, Mastny, S. 481-504. 
möglichen Zeitpunkt verständigt hatten, seine Bereitschaft zur weiteren deutschlandpolitischen Zusammenarbeit mit den Westmächten signalisiert zu haben schien. Mit Beginn des neuen Jahres wurde der Konsens aber gleich von mehreren Seiten herausgefordert. Da war zunächst die unübersichtliche Lage, die sich in Italien nach dessen Kapitulation herausgebildet hatte und aus der vor allem die italienischen Kommunisten Kapital zu schlagen vermochten. Mit zunehmender Besorgnis beobachtete das Foreign Office die Entwicklung und rätselte über die Intentionen Moskaus im Mittelmeerraum. Zugleich mehrten sich jetzt in London die skeptischen Stimmen hinsichtlich einer erfolgreichen Fortsetzung der Zusammenarbeit mit Moskau.

Aber auch von außerhalb verstärkte sich der Druck auf das Foreign Office, die Prämissen seiner Rußlandpolitik zu überdenken. Da nahm zunächst Paul Henri Spaak die Fäden jener Initiative auf, die schon im Winter 1941/42 zu Sondierungen von Vertretern nord- und westeuropäischer Randstaaten in London hinsichtlich eines atlantischen Sicherheitssystems geführt hatte. Im März 1944 unterrichtete er Eden vom Abschluß des belgisch-niederländischen Finanzabkommens, dem der Aufbau einer Zollunion der Benelux-Länder folgen sollte ${ }^{26}$. Für Spaak stellte sich nun die Frage, wie London eine mögliche Erweiterung dieses Kerns zu einem westeuropäischen Block beurteilte. Daß die Ursache dieses Vorstoßes in der Besorgnis lag, mit der er die Entwicklung in Osteuropa beobachtete, verdeutlichte er in einer Denkschrift, die er einen Tag nach dem Gespräch seinem britischen Amtskollegen übergab ${ }^{27}$. Die Situation, die bislang eine außenpolitische Zurückhaltung Londons gegenüber Kontinentaleuropa und ein Zusammengehen mit dem Kreml legitimiert hatte, war in seinen Augen überholt. Der russisch-tschechoslowakische Beistandspakt vom Dezember 1943 und der Hinweis des von den Briten bereits im Juli 1940 anerkannten Führers der tschechoslowakischen Exilregierung, Eduard Benesch, der Vertrag stehe auch für Polen und andere osteuropäische Länder offen, deuteten nach Meinung Spaaks darauf hin, daß die Sowjetunion nicht auf eine Lösung für Gesamteuropa wartete, sondern die Formierung Osteuropas unter russischer Führung anstrebte. Auch die Möglichkeit, das Sicherheitsproblem durch Integration der deutschen Wirtschaftskraft zu lösen, schien ohne britische Teilnahme in Frage gestellt. Spaak sah daher in der Bereitschaft Großbritanniens, sich den Bemühungen der Benelux-Staaten und Frankreichs um die wirtschaftliche und militärische Konsolidierung Westeuropas anzuschließen, einen ebenso konsequenten wie wünschenswerten Schritt, die Sicherheit und Prosperität gerade der kleineren europäischen Anrainerstaaten zu garantieren. Aus deren Sicht bot eine Anlehnung an die Europa vorgelagerte Inselmacht Schutz vor einem Ausgreifen des östlichen Blocks wie vor einer erneuten Hegemonie Deutschlands, vermochte daneben aber auch einer Blockdominanz durch Frankreich vorzubeugen ${ }^{28}$.

Wie zu erwarten, zeigte sich Eden skeptisch und warnte den Belgier vor einer Zweiteilung Europas. Der Außenminister erklärte, Vorschläge für einen Zusammenschluß der westeuropäischen Staaten müßten gegen die Notwendigkeit der Fortsetzung der Allianz mit Moskau während des Krieges abgewogen werden ${ }^{29}$. Genauer wurde der Außenminister gegenüber seinen Kabinettskollegen, denen er das Spaak-Memorandum zustellte,

${ }^{26}$ Zum Hintergrund vgl. Spaak, S. 111-116; sowie insgesamt Young, S. 7-16, besonders S. 12, 14.

${ }_{27}$ CAB 66/48, WP (44) 181, Annex: Translation of Memorandum by M. Spaak, 24.3.1944; Wood-

ward, British Foreign Policy, Bd. 5, S. $182 \mathrm{f}$.

${ }^{28}$ Vgl. dazu auch Young, S. 14.

${ }^{29}$ Woodward, British Foreign Policy, Bd. 5, S. $181 \mathrm{f}$. 
ohne daß freilich eine Erörterung der Minister folgte. In seinem Begleitschreiben führte Eden aus, daß die Formierung zweier Blöcke unter russischem bzw. britischem Einfluß zu gefahrvoll sei. Er verwies darauf, daß dadurch ein wirkungsvolles Vorgehen gegen Deutschland verhindert und der ehemalige Aggressor sich zum Zünglein an der Waage entwickeln würde. Eine Politik des Wettbewerbs um Deutschland, die als zentrifugale Kraft im Verhältnis beider Blöcke wirken und das Land in eine machtvolle Mittelposition versetzen mußte, war daher seiner Meinung nach unter allen Umständen zu vermeiden $^{30}$. Mit dem Versprechen an den belgischen Außenminister, ihm noch eine ausführliche Note über die britische Position zukommen zu lassen, sollte für das Foreign Office freilich die Angelegenheit nicht erledigt sein.

$\mathrm{Da}$ nun auch grundsätzliche Meinungsverschiedenheiten mit den militärischen Planern erwartet werden durften, deuteten die Diskussionen an, die im Post-Hostilities Planning Sub-Committee Anfang 1944 über die militärischen Aspekte einer Sicherheitsorganisation geführt wurden. Zum Entsetzen des Vorsitzenden Gladwyn Jebb ließen die Vertreter der Militärs nicht den geringsten Zweifel daran, daß sie von seiner „VierMächte-These" nichts hielten. Statt dessen erwarteten sie eine sowjetisch dominierte Einflußsphäre, innerhalb derer alle Sicherheitsbelange separat unter Führung Moskaus geregelt werden würden. Daraus zogen sie die Konsequenz, daß die Sicherheit des Westens durch die Fortsetzung der militärischen Kooperation mit Washington in der Form der Vereinigten Stabschefs garantiert werden sollte. China, notierte Jebb, war für sie „sowieso nur ein Witz" ${ }^{31}$.

Hinter dieser nüchternen Lagebeurteilung der militärischen Führung verbarg sich kaum weniger verhüllt als bei den Beamten des Außenministeriums die Angst vor einem Positionsverlust der eigenen Rolle innerhalb des Entscheidungsprozesses in London wie auch gegenüber den amerikanischen Stabschefs. War man allerdings im Foreign Office weitgehend überzeugt, daß ein Lösungsmodell die Kooperation mit Moskau einschließen mußte, so erblickten die Stabschefs umgekehrt im Prinzip der Polarisation, das ihnen den Fortbestand der Combined Chiefs of Staff, d. h. der anglo-amerikanischen Zusammenarbeit in verteidigungspolitischen Fragen, verbürgte, eine Möglichkeit zur Bewahrung der militärischen Vorrangstellung Großbritanniens. Eine neue Frontstellung versprach darüber hinaus auch hinsichtlich künftiger Bedarfsplanungen die Rettung eines guten Teils jenes Einflusses, den ihnen der Krieg beschert hatte. Die sich hier gegenüberstehenden militärischen und politischen Planungsansätze, mochten sie auch das gleiche Ziel im Visier haben, spiegelten also doch unterschiedliche (Eigen-)Interessen wider, und die Unvereinbarkeit der Ansätze, die zu einer unterschiedlichen Rußlandperzeption führte, ließ für die Zukunft Konflikte zwischen den beiden Entscheidungsträgern erwarten.

Bedenken hinsichtlich der Prämissen der britischen Außenpolitik äußerten neben Spaak und den Chiefs of Staff schließlich auch die Dominions. Am 20. März 1944 übermittelte der südafrikanische Premierminister Smuts Churchill und Eden ein Schreiben, in dem er das Außenministerium darum bat, für die bevorstehende Konferenz der Regierungschefs der Dominions eine Vorlage über die Wurzeln der russischen Politik und

\footnotetext{
${ }^{30}$ CAB 66/48 WP (44), 181, Memorandum by the Secretary of State for Foreign Affairs, „Future of Europe".

${ }^{31}$ FO 371/40740/U 1751, Minute Jebb, 19.2.1944.
} 
die sich daraus ergebenden Vor- und Nachteile der Schaffung einer regionalen, um Großbritannien herum aufgebauten westeuropäischen Organisation auszuarbeiten ${ }^{32}$. Vor allem mit seinem Hinweis auf die traditionellen Antriebskräfte des Kreml brachte Smuts eine neue Perspektive in die Diskussion. Ganz ein Diplomat alten Schlags, glaubte er die Beamten Whitehalls daran erinnern zu müssen, daß vieles bei der Neuordnung Europas von der traditionellen Politik der europäischen Mächte abhängen werde. Da seiner Meinung nach die langfristigen Ziele nationaler Politik einen nahezu konstanten Faktor darstellten, könne eine Analyse der früheren russischen Außenpolitik einigen Aufschluß über das zu erwartende Verhalten Moskaus bringen. Smuts ließ keinen Zweifel daran, daß eine „realistische“ Einschätzung der sowjetischen Intentionen die Grundlage für eine adäquate Sicherheitspolitik Großbritanniens in Europa bilden müsse, von der wiederum die Zukunft des Britischen Empire, insbesondere Indiens, des Mittleren Ostens sowie Mittel- und Südosteuropas abhing. Der Südafrikaner warnte dabei ausdrücklich vor der Möglichkeit eines geschlagenen und kommunistischen Deutschlands, das sich freiwillig dem sowjetischen Einflußbereich anschloß - „an eventuality more to be dreaded than anything else for the future of Europe and the world"33.

Smuts' Forderung nach einer historischen Aufarbeitung der russischen Außenpolitik stand in enger gedanklicher Nähe zu jenen Überlegungen, die George F. Kennan wenige Monate später nach seiner Versetzung an die Moskauer US-Botschaft zu entwickeln begann. Tatsächlich war der südafrikanische Premierminister davon überzeugt, daß ein Blick auf die Geschichte Anlaß zu Pessimismus gab. Im Gegensatz zu Spaak, der die anglo-sowjetische Allianz nicht ausdrücklich in Frage stellte, sondern für den Fall einer kooperationsbereiten Sowjetunion eine westeuropäische Organisation innerhalb einer von den Großmächten anvisierten Weltorganisation anstrebte, schwebte Smuts offensichtlich die Bildung eines regionalen Blocks als Gegengewicht zu möglichen Expansionsbestrebungen Moskaus vor. So jedenfalls wurde seine Initiative auch im Foreign Office verstanden, wie sich wenig später zeigen sollte. Smuts' Hoffnung auf eine Diskussion dieses Problems auf der Grundlage eines entsprechenden Memorandums des Außenministeriums erfüllte sich freilich nicht ${ }^{34}$.

Unter dem Eindruck dieser von verschiedenen und sehr unterschiedlichen Interessen gespeisten Initiativen war es erneut Gladwyn Jebb, der im Außenministerium mit der Aufgabe betraut wurde, die Umrisse einer Antwort auf die Initiative Spaaks auszuarbeiten, die nach Möglichkeit die geäußerten Befürchtungen entkräftete. Der Sicherheitsaspekt stand wie erwartet im Mittelpunkt erster, Anfang Mai 1944 vorgelegter Ausfüh-

32 FO 371/42677/W 4373, Smuts an Churchill und Eden, 20.3.1944; Woodward, British Foreign Policy, Bd. 5, S. 183.

${ }^{33}$ Ebenda. Vgl. auch Smuts' Rede „Thoughts on the New World“, die er am 25.12.1943 in London hielt und in der er für den Aufbau eines westeuropäischen Regionalpakts eintrat, um so die Ressourcenschwäche des Landes kompensieren zu können. Rede in: The Times, 3.12.1943; vgl. zur Wirkung der Rede auf das Außenministerium: Brief for United Kingdom Delegation at Terminal [Potsdamer Konferenz] "The ,Western Group' and Franco-British Treaty", 10.7.1945, in: DBPO I/1, S. 235-241, hier: S. 237.

${ }^{34}$ Zur Konferenz der Premierminister der Dominions Anfang Mai 1944 vgl. den Tagebucheintrag Edens am 4.5.1944, der die uneingeschränkte Zustimmung der Staatsoberhäupter zur britischen Außenpolitik ("not in a passive sense but with vigorous active approval") festhielt: „All this in marked contrast to 1937 and I admit that I find it very gratifying." Eden, The Reckoning, S. 442. 
rungen $^{35}$. Jebb hielt darin an Edens bereits gegenüber Spaak geäußertem Standpunkt fest, daß ein nicht expressis verbis gegen Deutschland gerichteter Block westeuropäischer Staaten von Moskau zwangsläufig als feindlich eingestuft werden würde, dies letztlich aber auf die Teilung Europas in eine britische und eine russische Einflußsphäre und den konkurrierenden Kampf um die Einbeziehung Deutschlands hinauslaufe. Bezüglich der eigenen Machtbasis für eine Führungsrolle offenbar verunsichert, glaubte er aber in einem europäischen Engagement Großbritanniens funktionale Aspekte zu erkennen, die dem Land zum Vorteil gereichen könnten. Ein engeres Zusammengehen mit den kontinentalen Nachbarn, so argumentierte er, könne grundsätzlich zur Stärkung der britischen Machtposition gegenüber den USA und der UdSSR wie auch gegenüber den Dominions führen. Dort, wähnte Jebb, mache sich nämlich Zweifel breit über den Willen und die Fähigkeit Großbritanniens, einen angemessenen Beitrag zur Schaffung einer Sicherheitsorganisation als eine der drei führenden Mächte zu leisten. Diesen Zweifeln konnte in seinen Augen entgegengetreten werden, wenn es gelang, nach außen durch ein Bündnis mit den westeuropäischen Staaten glaubhaft zu versichern, daß sich die strategische Position des Inselreichs verbessert und die materielle Basis seiner Verteidigungspolitik ausgeweitet hatte. Jebb nahm deshalb ausdrücklich Bezug auf die Idee des „,burdensharing“, von der sich das kriegsgeschwächte Land langfristig Entlastung zugunsten seiner generellen machtpolitischen Stellung erhoffen konnte: „while we were prepared at first to carry a heavy burden, other States should share this burden as they recovered their strength." 36

Jebbs Ausführungen können exemplarisch für die Tendenz des Außenministeriums stehen, Machterhaltungsaspekte in den Vordergrund der Planungen zu stellen. Einzelaspekte wie der von Spaak und Smuts vorgeschlagene Aufbau eines Regionalblocks sowie die Frage der Zerstückelung und Entmilitarisierung Deutschlands wurden auf ihren Funktionswert hin überprüft und bisweilen sogar im Sinne einer Kompensation der entstandenen materiellen Lücken zu instrumentalisieren versucht. Konfliktmodelle, wie sie offensichtlich die militärischen Planer anzustrengen begannen, blieben dabei ebenso unberücksichtigt wie eine konsequente Anlehnung an ökonomische und wirtschaftspolitische Leitlinien. Freilich ist ebenso offensichtlich, daß sich unter den Beamten mehr und mehr Zweifel hinsichtlich der wirtschaftlichen Fundierung ihrer machtpolitischen Ansprüche einstellten. Die Tatsache, daß hier nach Mechanismen gesucht wurde, die voraussichtlich die Schwächen der Inselmacht auszugleichen und so eine Fortführung ihrer friedensbewahrenden Großmachtrolle zu ermöglichen vermochten, ist sprechendes Indiz für ein verbreitetes Krisenbewußtsein in den Reihen des Foreign Office. So gesehen, machte sogar ein kontinentaleuropäisches Engagement Londons auf der Basis von „burden-sharing“ Sinn, zumal wenn ein westeuropäischer Block gegen Deutschland gerichtet war und damit die Prämisse der anglo-sowjetischen Kooperation nicht in Frage gestellt wurde.

Jebb sprach diese Zusammenhänge noch direkter als in seinen ersten Bemerkungen in einem weiteren Memorandum aus, das er ausschließlich den europapolitischen Proble-

\footnotetext{
35 FO 371/40692/U 4105, Memorandum Jebb, „Western Europe“, 9.5.1944; Woodward, British Foreign Policy, Bd. 5, S. $183 \mathrm{f}$.

${ }^{36}$ Ebenda.
} 
men widmete ${ }^{37}$. Darin warnte er vor einem kontinentaleuropäischen Alleingang ohne britische Beteiligung, der nicht nur Gefahr lief, über kurz oder lang von Deutschland dominiert zu werden, sondern aus seiner Sicht auch der britischen Reputation als Führungsmacht schaden und überdies in Struktur und Ausrichtung den außenpolitischen Primärinteressen Londons entgegenlaufen konnte. Nach Jebbs Vorstellung war ein westeuropäisches Sicherheitssystem unter britischer Beteiligung am ehesten auf dem Wege bilateraler Abkommen zu errichten, dessen Kern durch einen britisch-französischen Beistandspakt gebildet wurde. Ihm konnten weitere Abkommen mit den Niederlanden, Norwegen, Schweden und später Spanien, Portugal und Italien folgen. Jebb stellte als besonders vorteilhaft heraus, daß eine verteidigungspolitische Liaison mit Westeuropa gerade keine umfangreichen Verpflichtungen britischer Truppen auf dem Kontinent zur Folge hätte, sondern vielmehr zu einer Standardisierung der Ausrüstung führen würde, die auch der britischen Exportindustrie zugute kommen mußte, und zudem den Vorteil einer militärischen Zusammenarbeit mit den europäischen Staaten böte; nennenswerte Rüstungsvolumen waren zuvörderst in den britischen und französischen Kernlanden selbst zu schaffen. Es mochte fast schon an Donquichotterie grenzen, wie Jebb hier mit dem Gedanken operierte, die Einlösung des Sicherheitsanspruchs und die Stabilisierung der eigenen Machtposition könnten bei gleichzeitiger Reduzierung des Ressourceninputs erzielt werden. Ein wichtiger Erfolgsfaktor war in dieser Konstruktion natürlich der Kreml, der, so unterstellte Jebb, gegen eine regionale Allianz der westeuropäischen Staaten nichts einzuwenden haben werde, solange die Frontstellung gegen Deutschland deutlich zum Ausdruck komme und Moskau gleichzeitig die Chance erhalte, Osteuropa in einem komplementären Block mit gleicher Stoßrichtung zusammenzufassen.

Der konzeptionelle Widerspruch, der sich aus dem Anspruch auf eine Stabilsierungspolitik gegenüber Deutschland und dem Fortbestand der Kriegskoalition ergab, blieb offensichtlich zu diesem Zeitpunkt unerkannt. Symptomatisch hierfür war auch, daß das Foreign Office einen Vorschlag des ohnehin in den Kategorien einer Einflußsphärenpolitik denkenden Churchill vom 8. Mai 1944 ablehnte, mit dem der Premierminister gegenüber den Regierungschefs der Dominions die Schaffung regionaler Zusammenschlüsse, im Falle Europas einen „Europarat“, anregte; das Britische Empire sollte zu diesen Regionalpakten ebenso wie zu einem amerikanischen Block eine enge Verbindung eingehen. Eden gab zu bedenken, eine solche Organisation könne die UdSSR provozieren, und setzte sich schließlich seinerseits mit dem Vorschlag durch, innerhalb der Weltorganisation eine Art „UN-Kommission für Europa“ zu schaffen, die freilich in seinen Augen primär ein Mittel „for clearing up the war" darstellte und ausdrücklich als „Brücke“ zwischen Ost- und Westeuropa die Zielsetzungen des britisch-sowjetischen Vertrags unterstützen sollte; ein Weiterbestehen der Kommission nach Beendigung der unmittelbaren Aufbauarbeiten sah der Entwurf des Außenministeriums nicht vor ${ }^{38}$. Hinsichtlich der Schlußfolgerungen von Smuts notierte Orme Sargent: „The connexion which Field-Marshal Smuts had in mind was evidently the connexion between the need for curbing the Soviet Government's power in Europe and a means of doing so by set-

37 FO 371/40692/U 4366, Memorandum Jebb, „British Policy Towards Europe“, Mai 1944; Woodward, British Foreign Policy, Bd. 5, S. $184 \mathrm{ff}$.

${ }^{38}$ Vgl. Woodward, British Foreign Policy, S. 454-456. Vgl. auch Gilbert, Road to Victory, S. 768 f. 
ting up a Western regional bloc. This, of course, would be a completely new approach to the question, whereas the present memorandum definitely examines the matter from the point of view of fitting a regional system within the framework of a World Organisation, which is to be based on the principle of Anglo-Soviet co-operation. [...] All the same, in practice this regional system might very well, in altered circumstances, develop into a bulwark against Russian penetration, even though Anglo-Soviet co-operation still continued to operate as far as the subjugation of Germany was concerned." 39

Jebb wurde schließlich gebeten, seine beiden Memoranden zu einer Vorlage für die Chiefs of Staff und das APW-Komitee zusammenzufassen. Nach deren Placet war beabsichtigt, sie der britischen Delegation als Grundlage für Gespräche im Rahmen der Konferenz in Dumberton Oaks (21. August bis 28. September 1944) zu übergeben. Die am 20. Juni 1944 dem Außenminister vorliegende Denkschrift umriß noch einmal in konziser Form das Anliegen des Foreign Office und fügte hinzu, daß die britische Europapolitik auf keinen Fall auf Kosten der Übersee-Verpflichtungen gehen dürfe, sondern die traditionelle gleichgewichtige Interessenaufteilung britischer Politik zwischen Europa und Übersee unter besonderer Berücksichtigung des Verhältnisses zu den USA weiterhin oberster Grundsatz britischer Außenpolitik bleiben müsse ${ }^{40}$. Die Initiative der Beamten, ihre Überlegungen in Instruktionen für die Dumberton Oaks-Delegation umzumünzen, scheiterte freilich an der Intransigenz der Stabschefs, die erst Ende Juli zu einer Antwort bereit waren. Die dilatorische Behandlung dieses Planungsvorstoßes deckte sich daher auch mit dem Desinteresse Churchills und Edens an der Konferenz. Denn beide Minister waren stärker am Fortbestand der Führungsrolle Großbritanniens innerhalb eines starken Commonwealth und Empire sowohl gegenüber Moskau als auch gegenüber Washington interessiert ${ }^{41}$.

\section{Auseinandersetzungen zwischen Stabschefs und Außenministerium}

Die sich bereits im Frühjahr ankündigenden Meinungsdifferenzen mit den Militärs hatten sich in der Zwischenzeit verschärft. Von besonderer Bedeutung hierfür war die Umgestaltung der militärischen Planungsorganisation Anfang April 1944, die eine stärkere Verknüpfung langfristiger Strategie- und Rüstungsfragen mit den bisher im Vordergrund stehenden Problemen der unmittelbaren Besatzungsvorbereitung ermöglichte. Parallel zur Gründung des EIPS entstand als militärisches Gegenstück ein Post-Hostilities Planning Staff (PHPS), der direkt aus dem Post-Hostilities Planning Sub-Committee der Chiefs of Staff hervorging und der veränderten strategischen Lage in der Folge von „Overlord“, der Landung alliierter Streitkräfte in der Normandie am 6. Juni 1944, Rechnung tragen sollte. Zugleich reflektierte die Umbildung den Wunsch der Teilstreitkräfte, das Außenministerium von einer übermäßigen Einflußnahme fernzuhalten ${ }^{42}$. Gleichwohl blieb Jebb der Repräsentant des Außenministeriums; neben ihm waren noch

\footnotetext{
39 FO 371/40692/U 4105, Minute Sargent, 6.5.1944.

40 Vgl. Woodward, British Foreign Policy, Bd. 5, S. 189.

${ }^{41}$ Hathaway, S. 41.

${ }^{42}$ Gorst, S. 91-108, hier: S. 94.
} 
je ein Vertreter der drei Teilstreitkräfte sowie ein Deputy Director Civil Affairs vertreten. Ihre Aufgabe bestand erstens in der Ausarbeitung von Studien zu militärischen Problemen der Nachkriegszeit und zweitens in der Vorbereitung von Richtlinien für die mit den Waffenstillstandsverhandlungen betrauten Organe. Es war ausdrücklich erwünscht, daß das Komitee Kontakt zum Wirtschaftsplanungsstab hielt ${ }^{43}$. Mit dem Post-Hostilities Planning Sub-Committee wie jetzt auch mit dem PHPS waren daher die militärischen Nachkriegsplanungen aus der bestehenden Planungsorganisation der Teilstreitkräfte und ihren voraussichtlichen technischen, finanziellen und personellen Anliegen herausgenommen worden ${ }^{44}$. So ergab sich eine Stellung zwischen der militärischen Entscheidungsspitze der Chiefs of Staff einerseits, die keinen Hehl aus ihrem vorrangigen Interesse an der Kriegführung machte und deren Vorstellungen von einer Nachkriegsfriedensordnung sich auf den Fortbestand der anglo-amerikanischen Combined Chiefs of Staff sowie eine ausgeprägte Abneigung gegen eine Vier-Mächte-Regelung beschränkten, und den Beamten des Außenministeriums mit ihrem Primat der Wiederherstellung eines europäischen Mächtegleichgewichts andererseits.

Erste Schockwellen verursachte im Foreign Office eine Ende Mai 1944 vorgelegte Aufzeichnung, in der die Planer erste Überlegungen anstellten, von welchen Annahmen ihre Arbeit ausgehen sollte. Wie bereits festgestellt, hatte das Fehlen eindeutiger politischer Richtlinien bis jetzt jeden Ansatz zu einer geordneten Diskussion der anstehenden langfristigen sicherheitspolitischen Fragen sowohl im Military Sub-Committee als auch im Post-Hostilities Planning Sub-Committee vereitelt. Eine Richtlinienentscheidung war aber um so dringlicher, als mit der Gründung des PHPS kurzfristig anfallende Besatzungsprobleme zwar weiterhin erörtert werden mußten, nun aber ausdrücklich weitergehende Strategiestudien ins Zentrum der Planungstätigkeit rückten. In einem ersten, noch im Außenministerium entstandenen Vorentwurf bekräftigten die Beamten die Bedeutung einer Transformation der Kriegskoalition als Basis einer neuen „Weltsicherheitsorganisation"; im gleichen Atemzug schnitten sie aber die seit einigen Monaten schwelende Frage an, welche Konsequenzen sich für Großbritannien aus einer Frontstellung gegen die Sowjetunion ergäben, und rieten, als Vorbeugemaßnahme ein ausreichendes Rüstungsniveau anzustreben ${ }^{45}$. In überarbeiteter Form gingen diese Gedanken schließlich in ein PHP-Memorandum ein und verstärkten damit den Interessenkonflikt $^{46}$. Der Grundgedanke des Entwurfs, eine Lösung der Sicherheitsfrage unter trilateralen Auspizien anzustreben, wurde auch jetzt noch nicht in Frage gestellt. Die unter dem Einfluß der militärischen Vertreter entstandene Fassung ließ jedoch keinen $Z$ weifel daran, daß nicht von einem wirtschaftlich, politisch und moralisch zerstörten Deutsch-

${ }^{43} \mathrm{Vgl}$. insgesamt CAB 80/43, COS (44) 59, Memorandum by the Vice Chiefs of Staff, „Post Hostilities Planning Organisation“, 1.4.1944; FO 371/40736/U 4896, PHP (44) 43 (Final), Report by the Post-Hostilities Planning Staff, „Internal Organisation“, 26.5.1944. Zur Gründung des PHPS vgl. auch Lewis, S. 71-74. Zu Zielsetzung, Arbeitsweise und Zusammensetzung des am 8. August 1943 gegründeten Post-Hostilities Planning Sub-Committee vgl. FO 371/40736/U 3499, PHP (44) 27 (Draft), „Report on the Work Done by the Post-Hostilities Planning Sub-Committee. August 1943-May 1944".

${ }_{44} \mathrm{Vgl}$. ausführlich Gorst.

45 FO 371/40740/U 4978, PHP (44) 12 (0) (Draft), „Basic Assumptions for Post-War Strategic Planning", 26.5.1944.

${ }^{46}$ CAB 81/45, PHP (44) 12 (0), „Basic Assumptions for Staff Studies“, 7.6.1944. Vgl. auch Ross; Lewis, S. 102 f.; Gorst. 
land, sondern nur von den beiden Flügelmächten USA und Sowjetunion nach dem Krieg eine Gefahr ausgehen könne; da aber ein Dissens mit Moskau wahrscheinlicher sei, müsse Großbritannien im Zustand erhöhter Verteidigungsbereitschaft bleiben.

Diese Andeutungen, die zunächst nur auf die Garantie eines ausreichenden Rüstungsniveaus nach dem Krieg abhoben und vor einer überhasteten Demobilisierung warnten, genügten, um Feuer an die Lunte zu legen. Am zurückhaltendsten äußerte sich Gladwyn Jebb. Er fürchtete, so teilte er nach Lektüre der Aufzeichnung mit, daß es unmöglich sein werde, die Teilstreitkräfte von der Gefahr für den Bestand der Anti-Hitler-Koalition zu überzeugen, die darin liege, von der Sowjetunion als potentiellen Feind zu sprechen, oder gar eine solche Annahme zum Ausgangspunkt von Nachkriegsplanungen zu machen ${ }^{47}$. Demgegenüber reagierten andere hochgestellte Beamte wie Warner und Sargent skeptisch, ja verärgert über das „impertinente" Vordringen in genuines Foreign Office-Gewässer. Für Sargent, den seine Kollegen wegen seiner internen Aktivitäten gegen Chamberlains Appeasement-Politik „Moley“ nannten, waren die Gedanken der Planer "tendentious and out of focus and in contradiction with the policy of H.M. Government as conducted by the Foreign Office". Die Schlußfolgerungen gingen seiner Meinung nach über den Zuständigkeitsbereich des Planungsstabs hinaus, „and in any case the views of the Service members on P.H.P. staff on what is essentially a political question cannot be expected to carry any particular weight with the Foreign Secretary“48.

Doch es fanden sich auch abweichende Stimmen, die vor allem wegen ihres direkten Rückgriffs auf die traditionelle britische Verteidigungspolitik erwähnenswert sind. $\mathrm{Na}$ mentlich Frank Roberts und R. M. A. Hankey glaubten, vor einer Neuauflage der so verhängisvollen „Zehn-Jahres-Regel“, die die strategische Diskussion der Zwischenkriegszeit entscheidend dominiert hatte, warnen zu müssen. Er fühle, kommentierte Roberts seine Lektüre, daß die militärische Planung Moskaus ausschließlich unter dem Gesichtspunkt der eigenen Sicherheitsinteressen erfolge und nicht vom Fortbestand freundschaftlicher Beziehungen zu Großbritannien oder einem anderen Land abhänge. In seinen Augen war es daher notwendig, sich vom gleichen Prinzip leiten zu lassen, wollte man nicht die alten Fehler wiederholen: „One of the main reasons for our unpreparedness before this war was the now notorious ten years laying down that it could be assumed as axiomatic that we were unlikely to be engaged in a war with any other major power within a period of ten years. The formula now suggested amounts in practice to very much the same thing, since the only other major powers left in the world after this war will be the U.S.S.R. and the U.S.A. and it is assumed as axiomatic that a close association amounting to an alliance will bind us to these Powers for ten years. "49

Diese Bedenken bestanden aber lediglich bei einer Minderheit im Außenministerium, die, wie Roberts, zudem erst am Anfang ihrer Karriere stand und deren Hauptvertreter im für Deutschland zuständigen und im Ruf einer eher „deutschfreundlichen“ Haltung

47 FO 371/40740/U 6253, Minute Jebb, 7.6.1944.

${ }^{48}$ FO 371/40740/U 6253, Minute Sargent, 9.6.1944. Zu Sargents Haltung gegenüber der UdSSR vgl. Rothwell, S. 90-100 passim.

${ }^{49}$ FO 371/40740/U 4379, Minute Roberts, 26.5.1944; Minute Hankey, 27.5.1944. Vgl. auch Lewis, S. $101 \mathrm{f}$. Zur „Ten Year Rule“ vgl. die Diskussion bei Meyers, Britische Sicherheitspolitik, S. 388397; vgl, auch Taylor, S. 291-297. 
stehenden Central Department arbeiteten ${ }^{50}$. Ein Vermittlungsversuch Jebbs zwischen Außenamt und Stabschefs im Verlaufe eines „Off the record“-Treffens blieb ohne Erfolg. Die Chiefs of Staff erwiderten, daß auch über die britischen Sicherheitsinteressen nachgedacht werden müsse, „im Falle, daß alles schiefgeht“. Die Kluft zwischen den Beteiligten wurde freilich erst richtig deutlich, als sie Jebb belehrten, daß die rein strategischen Planungen nicht unnötig von politischen Erwägungen beeinflußt werden dürften. Dabei zeigte sich, daß sie in ihrer Haltung auch von dem Gedanken an die Sicherstellung ihres voraussichtlichen finanziellen, personellen und technischen Bedarfs nach dem Krieg geleitet wurden. "I may say", notierte Jebb anschließend, "that the Vice-Chiefs themselves showed no dispositon to want to plan operations against the Soviet Union but were merely concerned lest economy and popular outcry might in the long run succeed in reducing our armed forces below what was required for the protection of our vital interests, including of course in the first instance the control of Germany and Japan." ${ }^{11}$ Angesichts solch fundamentaler Interessengegensätze war ein Konflikt unvermeidlich, obwohl der PHPS, wie bereits festgestellt, bis zu diesem Zeitpunkt am Prinzip kollektiver Sicherheit unter sowjetischer Beteiligung nicht gerüttelt hatte und eine Basis für gemeinsame Planungen damit durchaus vorhanden schien.

Der Konflikt entzündete sich schließlich an einem Memorandum über Sicherheit im Nordatlantik und Westeuropa vom Juli 1944, in dem eine thematische Brücke zu dem eigentlichen deutschlandpolitischen Kern der Auseinandersetzung geschlagen wurde ${ }^{52}$. Die Studie war ein Auftrag des Außenministeriums an die Chiefs of Staff, die ihn ihrerseits an den PHPS weiterleiteten. Komplementär zu zwei Arbeiten über Sicherheit im Mittelmeerraum und im Fernen Osten sollte sie der Frage nachgehen, „what measures should be taken in the post-war world to secure British interests in the North Atlantic and Western Europe“. Die Stabschefs gaben ausdrücklich vor, sowohl die Möglichkeit einer Fortsetzung der Kriegsallianz wie auch die ihres Scheiterns in die Überlegungen

${ }^{50}$ Vgl. z. B. den Hinweis im Tagebuch Daltons: „G[ladwyn] J[ebb] says that in the F.O. there is opposition to his policy - the Four Power Plan - from, the Wilhelmstrasse', i.e. the Central Department. Here there is still high regard for German greatness, past and future, and for the legend of Bismarck. [Roger] Makins [1941/42 Leiter des Central Department] is still away but is returning soon, and little [Frank] Roberts, his No. 2, is anxious to ,keep everything all right for dear Roger'“" BLPES, Dalton Diaries I/27, fol. 154 (Eintrag 4.12.1942).

${ }^{51}$ FO 371/40740/U 6254, Minute Jebb, 15.6.1944. Für die These, daß hinter dem „worst case scenario “ der militärischen Planer das Interesse stand, die Stellung der Streitkräfte und die Einflußmöglichikeiten ihrer Entscheidungsspitzen auf die Mittelzuteilung nach dem Kriege abzusichern, spricht etwa auch die Tatsache, daß nach Vorlage einer vorläufigen Endfassung des PHPS-Papiers im September das Außenministerium postwendend mit einem Planungspapier über den zukünftigen Umfang der Streitkräfte konfrontiert wurde. Vgl. FO 371/40740A/U 7619, PHP (44) 23 (0) (Draft), „Procedure for Assessing the Size of Post-War Forces", 16.9.1944. Für einen weiteren Hinweis vgl. British Museum, Additional Manuscripts (im folgenden BM Add Mss) 52577, Cunningham Papers, Diaries, 1.6.1944.

52 FO 371/40741/U 6792, PHP (44) 17 (0) Final, Report by the Post-Hostilities Planning Staff, „Security in Western Europe and the North Atlantic“, 20.7.1944. Vgl. zum Folgenden Lewis, S. 107122. 
einzubeziehen ${ }^{53}$. Erste Entwürfe, die den bisherigen zweigleisigen Kurs des PHPS fortsetzten, den Primat des Foreign Office nicht anzugreifen, gleichzeitig aber auf die Möglichkeit eines Dissenses mit dem Kreml hinzuweisen, fanden durchaus positive Aufnahme im Außenministerium ${ }^{54}$. Mit ihrer Endfassung setzten sich die Planer dann aber zwischen alle Stühle und wurden sowohl vom Außenministerium wie von den Stabschefs unter scharfen Beschuß genommen.

Der PHPS machte darauf aufmerksam, daß angesichts der technischen Fortentwicklung in der Luftverteidigung, insbesondere des Baus von Langstreckenraketen, Großbritannien zu einem Teil Kontinentaleuropas geworden war. Die traditionellen strategischen Ziele, die auf die Kontrolle der Seeverbindungen und die Verhinderung feindlicher Übergriffe auf die Niederlande im wesentlichen beschränkt geblieben waren, bedurften daher einer dringenden Revision: Namentlich die Kontrolle des Luftraums und die Vereitelung von Angriffen einer feindlichen Macht auf kontinentaleuropäische Staaten waren als gleichberechtigte Ziele ins Kalkül zu ziehen. Wie, so lautete also die Frage, konnte die Sicherheit des Königreichs mit seinen weltweiten Verpflichtungen angesichts der drohenden wirtschaftlichen und personellen Engpässe unter den je unterschiedlichen Bedingungen eines feindlichen Deutschland, einer feindlichen Sowjetunion oder gar eines Zusammengehens beider garantiert werden? Für den Fall, daß es gelang, eine kollektive Sicherheitsorganisation aufzubauen, schlugen die Planer gegenüber Deutschland eine militärische Präventivpolitik vor, deren Kern in der Fähigkeit und der Bereitschaft lag, zusammen mit den westeuropäischen Alliierten gegen den Aggressor im Notfall mit militärischen Mitteln, insbesondere unter Einsatz der Luftwaffe, vorzugehen.

Sehr viel skeptischer wurden die Folgen einer Kollision mit Moskau und einer daran anknüpfenden verteidigungspolitischen Umorientierung skizziert. Angesichts der enormen geographischen Ausdehnung der Sowjetunion warnte der Planungsstab vor einer strategischen Überdehnung und stellte fest, daß bei dieser Eventualität ein britisches Engagement in Europa vor allem wegen der erforderlichen Ressourcen in Frage gestellt würde. Aus diesem Grunde betrachteten sie eine deutsch-russische Allianz als schlimmste aller Möglichkeiten. Eine Annäherung dieser beiden Staaten war aber nicht nur deshalb zu vermeiden, weil nach Meinung der militärischen Planer ein solches Bündnis selbst mit Hilfe der USA nicht zu schlagen sein würde, sondern weil im Falle einer Konfrontation mit Moskau Deutschland einen unverzichtbaren Verteidigungsbeitrag liefern und Erleichterung für Großbritanniens rüstungswirtschaftliche und strategische Überdehnungslage schaffen konnte: "It is an unpleasant fact that in the worst possible case if Russia does become hostile, Germany is the only country whose geographical position, manpower and other resources could provide the aid which might be essential to our

${ }^{53} \mathrm{FO} 371 / 40740 / \mathrm{U} 5909$, PHP (44) 17 (0) (T. of R.), „Security in the North Atlantic and Western Europe“, 19.6.1944. Einen ähnlichen Planungsauftrag hatten die Militärs vom Foreign Office bereits 1942 erhalten. Damals regte im Kontext von Stalins Forderung nach ungehinderter Durchfahrt zur Ostsee Roger Makins an, die damit verbundenen strategischen Folgen für Großbritannien von den Joint Planners erörtern zu lassen. Dabei sollten diese ihren Überlegungen die Hypothesen zugrunde legen „(a) of a hostile Germany with Russia (i) ally (ii) neutral (iii) hostile; and (b) of a hostile Russia with Germany neutral. I do not know whether we need consider the case of a hostile Russia with Germany allied“. Minute Makins, 4.5.1942, in: DzD I/3, S. 314.

${ }^{54}$ FO 371/40741A/U 6283, PHP (44) 17 (0) (Draft), 7.7.1944; Minute Roberts, 10.7.1944; Minute Warner, 12.7.1944; Minute Butler, 14.7.1944. 
preservation." 55 Wie zur Entschärfung dieser provokanten Aussage erklärten sie aber weiter, daß es geradezu eine Torheit wäre, durch einen Kurswechsel in der Deutschlandpolitik die Beziehungen mit Moskau zu gefährden, solange die britisch-russische Allianz fortbestehe. Es lag aus dieser Perspektive in der Konsequenz der Sache, daß Großbritannien angesichts seiner weltweiten Interessen und der beschränkten Ressourcen am meisten durch die erfolgreiche Umsetzung eines kollektiven Sicherheitsmodells mit dem Vereinigten Königreich, den USA und der Sowjetunion als Kern zu gewinnen hatte. In Ergänzung hierzu befürworteten sie ein regionales Verteidigungsbündnis der Länder Westeuropas, dem London durch die Bereitschaft zur Zusammenarbeit und das Versprechen militärischer Unterstützung im Ernstfall zur Seite stehen sollte.

Genau an dieser Schwachstelle hakten die Stabschefs ein, als sie das Memorandum auf ihrer Sitzung am 26. Juli 1944 einer vernichtenden Kritik unterzogen. Der PHPS, so monierten sie, "had failed to face up to the hard military facts of the problem" 56 . Die Eventualität eines Zusammenbruchs der Weltorganisation war in ihren Augen nicht gebührend berücksichtigt worden. Zwar treffe es zu, daß man sich der Kooperation der westeuropäischen Länder versichern müsse; jedoch werde der Eindruck vermittelt, daß ein regional begrenzter Zusammenschluß die Sicherheit des Großbritannien vorgelagerten Festlandes garantiere. Im Falle eines Scheiterns der Weltorganisation bildete ihrer Meinung nach ein isolierter Regionalblock nur den ersten Schritt auf dem Weg zu einem umfangreicheren Sicherheitssystem, "which, if the security of these Islands were to be secured, must include a part, if not the whole, of Germany". Deutschland, so unterstrichen sie, sei "the key to the security of these Islands in the future and, however unpalatable the fact might be, there might well come a time when we should have to rely on her assistance against a hostile Russia". Mit ihrer langfristigen Perspektive, die im Gegensatz zu dem aus ihrer Sicht lediglich die kurzfristigen Ziele in den Blick nehmenden Foreign Office-Konzept stand, banden die höchsten britischen Militärs das Problem der Sicherheit Großbritanniens unauflösbar an dessen Deutschlandpolitik. Sie betonten, daß von den Maßnahmen, die gegenüber Deutschland ergriffen werden sollten, auch dessen Fähigkeit zur Leistung eines Sicherheitsbeitrags und damit letztlich die Verteidigungsfähigkeit Großbritanniens abhänge. Insbesondere die Argumente für und gegen eine Teilung Deutschlands waren in diesem Lichte neu gegeneinander abzuwägen.

Die Brisanz dieses Befundes lag auf der Hand, und so konnte es kaum überraschen, daß die Kommentare der Stabschefs im Außenministerium Empörung auslösten ${ }^{57}$. Mit ihrer Absicht, die Bedeutung eines westeuropäischen Verteidigungsbündnisses unter Hinweis auf eine unbedingte Einbeziehung des deutschen Potentials zu relativieren, widersprachen die Chiefs of Staff diametral dem Verlangen des Außenministeriums, eine sicherheitspolitische Konsolidierung Kontinentaleuropas auf regionaler Basis unter dem Schirm der Weltorganisation zu erreichen. Nur mühsam verbargen sie hinter ihren Empfehlungen die Abneigung gegen zu internationalistische Lösungsversuche der Sicherheitsproblematik. Aus der Sicht des Außenministeriums dagegen war es geradezu ein

${ }^{55}$ FO 371/40741/U 6792, PHP (44) 17 (0) Final, Report by the Post-Hostilities Planning Staff, „Security in Western Europe and the North Atlantic", 20.7.1944.

${ }^{56}$ FO 371/40741 A/U 6793, Record of Discussion of the Chiefs of Staff, 26.7.1944. Dort auch die folgenden Zitate.

57 FO 371/40741 A/U 6793, „Comments by the Chiefs of Staff on Policy Towards Western Europe“ (undatiert). 
Affront der bisherigen, während des gesamten Krieges gegenüber der Sowjetunion verfolgten Politik, wenn die Stabschefs den Gedanken eines deutschen Verteidigungsbeitrags ins Spiel brachten. Deren Hinweise auf den Gewinn an "strategischer Tiefe“, die durch eine Anbindung Deutschlands erreicht würde, sowie die personelle, finanzielle und industrielle Entlastung der eigenen Verteidigungsposition mochten zwar zutreffen; aber wurde hier nicht mindestens indirekt auch von einer militärischen Wiederbewaffnung Deutschlands gesprochen? Durfte man, noch bevor der jüngste Waffengang gegen die Deutschen erfolgreich zu Ende geführt war, selbst in Planspielen überhaupt den Gedanken ernsthaft diskutieren, sie erneut unter Waffen zu stellen? Die Bemerkungen der Stabschefs über den Zusammenhang der zukünftigen Deutschlandpolitik Londons mit der Sicherheit der britischen Inseln überschritten aus der Sicht des Außenministeriums zudem deutlich die Grenze der Zuständigkeit, und das in einer Weise, die massive Kritik an der bisherigen Haltung der Regierung zur Dismemberment-Frage vermuten ließ. Die Feststellung, daß, wie der First Sea Lord, Admiral Andrew Cunningham, nach der Sitzung lapidar in seinem Tagebuch festhielt, „[it] was generally agreed that Russia would be the only danger in the foreseeable future ${ }^{\text {"58, }}$, stellte eine Grundprämisse der bisherigen britischen Außenpolitik radikal in Frage. Die Haltung der Chiefs of Staff war deshalb zugleich Ausdruck von Kompetenzstreitigkeiten. Mit ihr verbanden sie den Wunsch, die militärische Planungsorganisation in den ausschließlichen Zuständigkeitsbereich der Militärs zurückzuführen ${ }^{59}$.

Jebb, der am Tage vor der Besprechung der Stabschefs noch die Meinung äußerte, daß die Studie des PHPS die deutschland- und rußlandpolitischen Aspekte im richtigen Verhältnis darstelle ${ }^{60}$, sprach für die Mehrheit der Beamten, wenn er der Kritik der Militärs entgegenhielt, daß eine Politik, die darauf abziele, seine Feinde gegen die eigenen Verbündeten aufzubauen, nur auf eine Art "suicidal mania“ hindeuten könne ${ }^{61}$. Eine abweichende Meinung äußerte hingegen der Leiter des Joint Intelligence Committee, William Cavendish-Bentinck: „I do feel that if we give the impression of persistently refusing to allow any mention that Russia may later on be non-co-operative and even hostile, we shall have in the Service Departments the same reputation vis-à-vis Russia as Sir Horace Wilson had as regards Germany from 1937 to the outbreak of the present war, with the result that they may tend to discount our opinions and regard us as being ostrich like so far as Russia is concerned." 62 Dessenungeachtet hatte Eden bereits in einer Sitzung des Armistice and Postwar Committee eine Woche vor dem entscheidenden Treffen der Stabschefs Kriegsminister Grigg sofort und energisch widersprochen, als dieser, wohl als eine Art Versuchsballon, die Minister über das Anliegen der Chiefs of Staff ins Bild setzte; dabei hatte der britische Außenminister die Mehrheit seiner Kollegen eindeutig hinter sich ${ }^{63}$. Offensichtlich sah er auch seine Strategie, die er in Gesprächen mit Spaak, Lie und dem Niederländer van Kleffens verfolgte, in Gefahr, sollten die Gedankenspiele der Militärs in die Presse gelangen. Ihnen gegenüber hatte er die abwartende Haltung der britischen Regierung in der Frage eines westeuropäischen Bündnisses nur eine Woche

58 BM Add Mss 52577, Cunningham Papers, Diaries, 26.7.1944.

$59 \mathrm{Vgl}$. die Hinweise bei Gorst, S. 97.

${ }_{60} \mathrm{FO} 371 / 40741 \mathrm{~A} / \mathrm{U}$ 6792, Minute Jebb, 25.7.1944.

${ }^{61} \mathrm{FO} 371 / 40741 \mathrm{~A} / \mathrm{U}$ 6793, Minute Jebb, 28.7.1944.

${ }_{62}$ FO 371/40741A/U 6791/748/G 70, Minute Cavendish-Bentinck, 18.7.1944.

${ }^{63}$ Tyrell, S. 447. 
zuvor noch mit dem Hinweis auf die bevorstehende Konferenz zur Gründung einer Weltorganisation entschuldigt und damit die Sicherheitsfrage erneut mit der Schaffung der Vereinten Nationen in Verbindung gebracht. Ein überhastetes Vorgehen spielte nach Edens Meinung auch nur den Isolationisten in den USA in die Hände, die einen westeuropäischen Pakt als Zeichen ansehen könnten, daß Europa der amerikanischen Unterstützung nach dem Krieg nicht bedurfte. Ihm selbst, so hatte er den Exil-Politikern erläutert, schwebe eine Art Neuauflage des Locarno-Paktes vor - jedoch ohne die Deutschen ${ }^{64}$. Schließlich war es besonders ärgerlich, daß die Militärs sich anschickten, auf der Basis ihrer sicherheitspolitischen Grundvorstellungen wichtige Aspekte der Deutschlandpolitik des Außenministeriums in Frage zu stellen und sich direkt in die laufenden trilateralen Verhandlungen zur „Dismemberment“-Frage einzumischen. Wie war diese diffizile Situation in beiderseitigem Einvernehmen zu lösen?

Angesichts der geschilderten Problemlage überrascht es nicht, daß trotz ihres allgemeinen Charakters die Entscheidung tatsächlich auf deutschlandpolitischem Gebiet fiel. Ende August 1944 legte der PHPS eine Studie zu den Vor- und Nachteilen einer Aufteilung Deutschlands aus militärischer Sicht vor ${ }^{65}$. Daß die Planer in ihr auch kurz auf die Verhinderung der deutschen Wiederbewaffnung, die als eines der beiden strategischen Primärziele definiert wurde, eingingen, war kaum mehr als ein argumentatives Feigenblatt, das sie zudem noch in ihrem Sinne zu benutzen wußten: Die Teilung Deutschlands, so ihre Schlußfolgerung, beuge auch der Gefahr einer deutschen Wiederbewaffnung vor, da sie die gemeinsame verteidigungspolitische Organisation der Teilstaaten verhindere und ein eventuelles Eingreifen der Alliierten erleichtere. Diese Bemerkungen vermochten jedoch kaum ihr eigentliches Anliegen zu verdecken: die Mobilisierung des deutschen Potentials zur Nutzung für eine antizipierte Frontstellung des Westens gegen die Sowjetunion und die Ausrichtung der britischen Deutschlandpolitik auf dieses Ziel hin. Ein feindliches Rußland, erklärten sie, erfordere die vollkommene Umorientierung der gegenwärtigen Verteidigungspolitik. Und in diesem Falle "we should require all the help we can get from any source open to us, including Germany“66. Die Militärplaner sprachen sich daher für eine Aufteilung Deutschlands aus, weil nur so die Verfügbarkeit über deutsche Ressourcen und eine wenigstens teilweise Wiederbewaffnung Deutschlands gesichert sei. Das Festhalten an Gesamtdeutschland würde keine deutschen Mittel bereitstellen, schon gar nicht für Verteidigungszwecke, weil man sich kaum mit Moskau werde einigen können. „Our interests are therefore likely to be better served in this event by the acceptance of dismemberment, for we might hope eventually to bring North-Western, and possibly Southern Germany also, within the orbit of a Western European Group. This would give increased depth to the defences of the United Kindgom and increase the war potential of that group. “67 Da überdies kaum zu erwarten war, daß ein einheitlicher deutscher Staat, noch dazu wiederbewaffnet, von Moskau toleriert werden würde - es sei denn, er befände sich unter dessen Einfluß -, bot die Teilung zugleich

${ }^{64}$ FO 371/40700/U 6468, Eden an N. Oliphant, N. Bland und L. Collier, 19.7.1944; BM Add Mss 56400, Harvey Papers, Diaries, 15.7.1944; Harvey (Hrsg.), S. 348. Vgl. Spaak, S. 119-121.

${ }^{65}$ FO 371/39080/C 11521, PHP (44) 15 (0), „Dismemberment of Germany“, 25.8.1944. Vgl. zum Folgenden Lewis, S. 122-135.

${ }^{66}$ Ebenda. In einer früheren Fassung folgte an dieser Stelle noch der Halbsatz „if the U.S.A. were not to come to our aid in time". Vgl. FO 371/39080/C 11062, Entwurf vom 17.8.1944.

67 FO 371/39080/C 11521, PHP (44) 15 (0), „Dismemberment of Germany“, 25.8.1944. 
die beste Möglichkeit, ein Bündnis Gesamtdeutschlands mit der Sowjetunion auszuschließen.

Die von den Stabschefs ausgeführten Argumente spiegelten sich direkt in der überarbeiteten Fassung der PHPS-Studie über Sicherheit in Westeuropa und dem Nordatlantik, die nun, da das Foreign Office in der Zwischenzeit seine Mitarbeit in dem Planungskomitee der Militärs aufgegeben hatte, schwerpunktmäßig den Fall einer feindlichen Sowjetunion untersuchte und das Memorandum über die Teilungsfrage in idealtypischer Weise ergänzte ${ }^{68}$. Jetzt wurde deutlich, daß der Überlegung der Planer, eine Aufteilung verhindere auch die Wiederbewaffnung Deutschlands, das Bemühen zugrunde lag, wichtige Zeit für den Aufbau einer westlichen Sicherheitsgemeinschaft gegen die Sowjetunion zu gewinnen. Sie gingen davon aus, daß für die kommenden zehn Jahre keine bewaffnete Auseinandersetzung mit Moskau bevorstand; währenddessen könne man die notwendige Revision der Strategie- und Bedarfspläne realisieren und durchaus eine Politik betreiben, die die Verhinderung der Remilitarisierung Deutschlands zum Ziel habe. Eine deutschlandpolitische Kursänderung war aber unabdingbar, sobald die UdSSR ihre Kooperationsbereitschaft aufgab, und daran zweifelten die militärischen Planer immer weniger. Ein deutscher Verteidigungsbeitrag hing dann nicht zuletzt von der Deutschland- und Besatzungspolitik der Alliierten nach Kriegsende ab; die Entscheidung in der Dismemberment-Frage hatte aus ihrer Sicht eine präjudizierende Wirkung auf den aller Wahrscheinlichkeit nach unabwendbaren militärischen Mobilisierungsprozeß der nichtkommunistischen Staaten. Daneben spielte die Geschwindigkeit eine Rolle, mit der es gelang, die Deutschen mit westlicher Unterstützung in der Zeitspanne zwischen einer deutschlandpolitischen Kursänderung und einem sowjetischen Angriff wiederzubewaffen. Mit unterschwelligem Bedauern konstatierten die PHPS-Planer, daß drastische Maßnahmen gegen Deutschland in der unmittelbaren Nachkriegsphase wohl kaum zu umgehen waren: „Experience, however, suggests that with our encouragement Germany could achieve a certain degree of rearmament with completely modern equipment within a comparatively short period after our change of policy. In any case, her airfields and ports would be of immediate value in resisting Soviet aggression. [...] Even if she were totally disarmed she would provide added depth to our defensive zone, airfields from which to exercise a threat to Soviet territory and ports to assist in the control of the exits from the Baltic. Her manpower and industrial resources would be of particular value if she had achieved some measure of rearmament." Großbritannien selbst - und hier wird erneut die eigene Sorge um die militärische Vorrangstellung des Landes und die Sicherstellung der dafür notwendigen rüstungswirtschaftlichen Ressourcen deutlich - müsse ein Rüstungsniveau anstreben, das im Verbund mit dem Potential der USA, deren enge Kooperation als lebenswichtig betrachtet wurde, der europäischen Verbündeten und (West-)Deutschlands eine militärische Überlegenheit gegenüber der Sowjetunion garantiere.

Die kritischen Reaktionen im Außenministerium beschränkten sich zunächst darauf, die in ihren Augen unlogische Gedankenführung des PHPS-Reports aufzuweisen. Schließlich unterbreiteten die Beamten ihre Sicht der Dinge in einem Gegenmemorandum, das Eden dem APW-Komitee unterbreitete. Es war fast zu erwarten, daß die Be-

${ }^{68}$ FO 371/40741A/U 7618, Revised Draft of PHPS Report „Security in Western Europe and the North Atlantic", 15.8.1944. Dort auch das folgende Zitat. 
amten die Auffassung, ein geteiltes Deutschland wirke der Gefahr seiner Wiederbewaffnung entgegen und sei im Bedarfsfalle besser in eine Frontstellung gegen eine neue Aggressormacht zu integrieren, als widersprüchlich und unrealistisch herausstellen würden. Der Gedanke, daß die Teilung die Deutschen gerade so lange niederhalten würde, wie es den britischen Interessen entsprach, nur um sie dann um so schneller in einen potenten Verbündeten im Kampf gegen eine feindliche östliche Mächtekonstellation zu

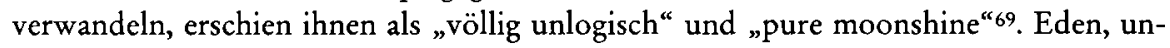
willentlich die Befürchtung Roberts und Hankeys hinsichtlich einer neuerlichen „TenYear-Rule" bestätigend, wandte gegen die Stabschefs ein, es sei unwahrscheinlich, daß es innerhalb der nächsten Jahre einen Grund für einen Krieg mit der Sowjetunion geben werde. In der Zwischenzeit basiere die Politik der Regierung Seiner Majestät auf einer anglo-sowjetischen Allianz, und man müsse alles unternehmen, um die Beziehungen mit Moskau zu verbessern. Den Vorschlag einer Revision der Deutschlandpolitik mit dem Ziel, einen Teil gegen die Sowjetunion zu mobilisieren, bezeichnete er als geradezu phantastisch („little less than fantastic“), ja noch schlimmer: „It is playing with fire.“ Seine Realisierung zerstöre das bestehende Bündnis mit der Sowjetunion und unterminiere die Wirksamkeit der Entmilitarisierungsmaßnahmen in Deutschland. „There is the strongest political objection to basing any of our plans for Germany on the possible need to use Germany one day as allies against an aggressive Russia. " 70 Nicht unbedeutende Schützenhilfe bekam das Foreign Office vom Economic and Industrial Planning Staff, der in einer Studie über die wirtschaftlichen Aspekte der Teilungsfrage zu einem negativen Urteil gelangte. Dismemberment mußte ihrer Ansicht nach die deutsche Wirtschaft schwächen, ihre Fähigkeit zur Leistung von Reparationen schmälern und in beträchtlichem Maße die alliierten Devisenkassen belasten ${ }^{71}$.

Die Karten waren also für Eden und seine Beamten günstig verteilt, als es am 4. Oktober 1944 zu einer Aussprache zwischen dem Außenminister, begleitet von Orme Sargent, und den drei Stabschefs kam. Niemand erwartete freilich eine Lösung des Konflikts, denn das Foreign Office befand sich in der stärkeren Position und sah sich kaum veranlaßt, Terrain preiszugeben. Beispielhaft für die Stimmung im Amt war sicherlich Wards Reaktion auf das Strategie-Papier des PHPS. Man sei für dessen Inhalt schließlich nicht verantwortlich, und im übrigen werde es durch die Vorlage der Aufzeichnung von Eden im Armistice and Postwar Committee mit Sicherheit torpediert. Nicht ohne Überheblichkeit drückte er seine Hoffnung aus, die Minister würden Entscheidungen treffen, „which would kill this P.H.P. paper on Western Europe by implication and relieve us

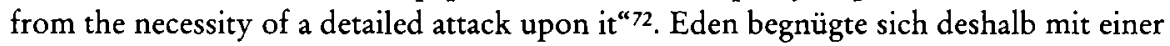
Wiederholung bekannter Vorwürfe und erinnerte die militärische Führung an die fehlgelaufenen Planungen nach dem Ersten Weltkrieg, als man in Frankreich den einzig ge-

${ }^{69}$ FO 371/39080/C 11955, Minute J. G. Ward, 28.8.1944; Minute Troutbeck, 24.8.1944.

$70 \mathrm{FO} 371 / 39080 / \mathrm{C} 13518$, Minute (unsigned), 4.10.1944; FO 371/39080/C 12806, Memorandum by the Secretary of State for Foreign Affairs, "The Dismemberment of Germany", 20.9.1944; teilweise abgedruckt in: Ruhrfrage, S. 257-261; FO 371/39080/C 12404, „The Dismemberment of Germany. Summary of Foreign Office Paper“, 6.9.1944 (Hervorhebung M. P.).

${ }^{71}$ FO 371/39080/C 12029, EIPS/P (44) 30 (Final), „Economic Aspects of the Proposal for the Dismemberment of Germany“, 2.9.1944 (auch in: FO 1005/959).

72 FO 371/40741A/U 7618, Minute J. G. Ward, 19.9.1944. Für Eden war die PHPS-Denkschrift „a terrible paper however it is looked at". Ebenda, Minute Eden, 23.9.1944. 
fährlichen Feind erblickte und damit den Planungen eine Fehleinschätzung zugrunde gelegt hatte - mit verheerenden Folgen ${ }^{73}$.

Die Stabschefs sahen auf der anderen Seite ebensowenig Grund zum Nachgeben. Zur Verteidigung ihres Anliegens schoben sie den Hinweis auf eine alle Eventualitäten einbeziehende Planungsarbeit vor. Im gleichen Atemzug warfen sie den Beamten vom Außenministerium Wunschdenken und Engstirnigkeit vor: „Such an ostrich like attitude“, kommentierte Cunningham fassungslos in seinem Tagebuch. „One cannot close one's eyes and by not thinking of it remove the potential danger of a hostile Russia. ${ }^{774}$ Das Foreign Office lasse sich offensichtlich von der gefährlichen Illusion leiten, daß die auf Kooperation mit dem Kreml unter dem Dach einer Weltorganisation abzielende Außenpolitik Londons „mit historischer Gewißheit" von Erfolg gekrönt sein werde und ein Nachdenken über die Konsequenzen eines möglichen Scheiterns überflüssig mache ${ }^{75}$.

Gegen diese Geschlossenheit der Stabschefs vermochte schließlich auch der Außenminister nichts auszurichten. Eine zugunsten der einen oder anderen Seite ausfallende Lösung war aussichtslos, und so einigten sich alle Beteiligten darauf, ihre jeweiligen Memoranden einstweilen zurückzuziehen. Die Chiefs of Staff verpflichteten sich zusätzlich zu einer besonderen Geheimhaltung im Umgang mit ihren Thesen ${ }^{76}$. Eine Entscheidung über die Basis künftiger Planungen war damit nicht gefallen. Vielmehr glaubte das Außenministerium, wie der Kommentar Wards zeigte, daß es gelungen war, die Häresien der Militärs wenn schon nicht zu eliminieren, so doch wenigstens schadensbegrenzend einzudämmen und auf den inneren Planungszirkel zu beschränken. Die vordergründigen Erfolge der Moskauer Außenministerkonferenz nur zwei Wochen nach Edens Treffen mit den Stabschefs und die Tatsache, daß der Kreml in der Teilungsfrage nach Jalta auf die britische Interpretation einschwenkte, schienen den Kurs des Außenministeriums zu bestätigen.

Dies bedeutete freilich nicht, daß das Eintreten des Außenministeriums für den Aufbau eines westeuropäischen Sicherheitsbündnisses in der Folgezeit die Form eines ausgearbeiteten Konzepts annahm und Eden gegenüber den andauernden Sondierungsversuchen insbesondere Spaaks die Initiative übernahm. Obwohl noch im Oktober 1944, also nach der Konferenz von Dumbarton Oaks, ein internes Treffen erneut bekräftigte, daß ein auf militärische und technische Vereinbarungen begrenztes Abkommen mit Frankreich und den Niederlanden wünschbar sei ${ }^{77}$, verhinderte letztlich die traditionelle Abneigung gegen ein kontinentales Engagement eine stringente Europapolitik. Zum einen stellte sich Churchill gegen die Foreign Office-Pläne und untersagte Eden, in Gespräche über ein Verteidigungsbündnis mit den westeuropäischen Staaten einzutreten; die Schwäche dieser Staaten und die zu erwartenden wirtschaftlichen Schwierigkeiten Großbritanniens, die den sparsamsten Einsatz der militärischen Ressourcen geboten,

${ }_{73}$ FO 371/39080/C 13518, Minute Sargent, 4.10.1944; Ruhrfrage, S. 264.

74 BM Add Mss 52577, Cunningham Papers, Diaries, 4.10.1944.

75 FO 371/39080/C 13518, Note by the Chiefs of Staff, "The Study of Post-War Problems", 2.10.1944; Ruhrfrage, S. 261-263.

${ }^{76}$ FO 371/39080/C 13518, Minute Sargent, 4.10.1944; FO 371/43336/N 6177, Minutes of Meeting, 4.10.1944; BM Add Mss 52577, Cunningham Papers, Diaries, 4.10.1944.

77 FO 371/40721/U 7956, Minutes of Meeting, 20.10.1944; vgl. auch Woodward, British Foreign Policy, Bd. 5, S. $192 \mathrm{f}$. 
machten in seinen Augen eine solche Anbindung zu einem Hasardspiel ${ }^{78}$. Zum anderen zeigte sich Eden selbst angesichts der präzisen Vorstellungen seines belgischen Kollegen unvorbereitet und überfordert und erging sich in allgemeinen und unverbindlichen Formulierungen ${ }^{79}$. Zugleich waren damit auch alle Bemühungen der Exilregierungen Frankreichs und der Benelux-Staaten, die im Winter 1943/44 intensiv an Plänen für eine engere wirtschaftliche Zusammenarbeit unter Einschluß der deutschen Ressourcen nach Kriegsende gearbeitet hatten, wirksam torpediert ${ }^{80}$. Die Gesprächsansätze verloren sich schließlich in der ersten Hälfte des neuen Jahres im Getriebe der Konferenzvorbereitungen für Jalta und Potsdam sowie dem Wahlkampf für die Parlamentswahlen.

Den Chiefs of Staff war es immerhin gelungen, eine Festlegung der Planungsrichtlinien durch das Außenministerium abzuwenden. Zwar hatten sie zugestanden, ihre Formulierungen künftig etwas behutsamer zu fassen. Doch sahen sie ihre Planungsaktivitäten, auf welchen Hypothesen sie diese auch immer gründen mochten, in keinster Weise eingeschränkt. Darüber hinaus war es ihnen gelungen, die militärische Planungsorganisation wieder weitgehend dem Einfluß des Foreign Office zu entziehen. Die zwischen Eden und ihnen getroffenen gegenseitigen Stillhaltezusagen, mit denen insbesondere der Außenminister möglichen innen- und außenpolitischen Schäden zuvorkommen wollte, ermöglichten auch den Militärs ein weitgehend störungsfreies, von politischen Aspekten gänzlich befreites Arbeiten unter ihren eigenen Prämissen. Sie blieben auch in der Folgezeit unbeeindruckt von den Argumenten der Foreign Office-Beamten ${ }^{81}$ und legten Mitte November 1944 sogar Endfassungen ihrer Memoranden zur Teilungsfrage - jetzt unter dem bezeichnenden Titel „Dismemberment of Germany - Military Aspects" 82 sowie zur Sicherheit in Westeuropa ${ }^{83}$ vor. Gleichzeitig begannen die Planungsarbeiten für ein globales Strategiepapier, das auf den Regionalstudien aufgebaut und schließlich im Juni 1945 abgeschlossen werden sollte ${ }^{84}$. Freilich wäre es verfehlt, aus der Rückschau gerade die Re-Monopolisierung der militärisch-strategischen Planungen als einen Erfolg der Stabschefs überzubewerten. Vielmehr mußten sich die Militärs angesichts der späteren Entwicklung die Frage stellen, ob nicht umgekehrt die erfolgreiche Abdrängung ih-

${ }_{78}$ FO 371/40723/U 8472 und FO 371/40723/U 8473, Minutes Churchill, 25.11.1944; vgl. auch Woodward, British Foreign Policy, Bd. 5, S. 193 f.; ders., British Foreign Policy in the Second World War, S. 464; Young, S. 14.

${ }^{79}$ Spaak, S. 119-125; Harvey (Hrsg.), S. 365 (Eintrag 11.11.1944).

${ }^{80}$ Young, S. 10-14. Die Block-Pläne fanden auch keine Befürworter auf amerikanischer und sowjetischer Seite. Während Moskau, wie von Großbritannien befürchtet, eine antisowjetische Ausrichtung argwöhnte, vermutete Washington dahinter einen Schritt in die Richtung eines europäischen Wirtschaftsblocks. Auch de Gaulle wandte sich ab, sah er doch eine für seine Großmachtpläne wichtigere Allianz mit Stalin zur Kontrolle Deutschlands in Gefahr. Vgl. ebenda, S. 13 f., 29.

${ }^{81}$ Vgl. z. B. BM Add Mss 52577, Cunningham Papers, Diaries, 12.10.1944; CAB 79/82, COS (44) 345, Meeting 23.10.1944.

82 FO 371/39080/C 16045, PHPS (44) 15 (0) (Revised Final), „Dismemberment of Germany - Military Aspects", 15.11 .1944 (auch in: CAB 81/45 und CAB 80/89, COS (44) 1012(0)); vgl. Woodward, British Foreign Policy, Bd. 5, S. $242 \mathrm{ff}$.

${ }^{83}$ FO 371/40741B/U 8181, PHP (44) 27 (0) (Final), "Security in Western Europe and the North Atlantic“, 9.11.1944.

${ }^{84}$ CAB 81/46, PHP (45) 29 (0) (Final), „The Security of the British Empire“, 29.6.1945. Vgl. dazu Lewis, S. 162-177. 
rer Planungen auf rein militärische Belange eine folgenschwere Verkürzung des tatsächlichen Sachverhalts bewirkte.

Die skizzierten Auseinandersetzungen wiesen in ihrem prägenden Einfluß auf die hier darzustellende Sicherheits- und Deutschlandpolitik Londons zwei Merkmale auf. Sie stellten erstens die Bedeutung des Faktors Moskau für die Formulierung der britischen Deutschlandpolitik heraus. Auch wenn im Anschluß an die Konferenz von Jalta ein langsamer Ernüchterungsprozeß im Außenministerium einzusetzen begann und man mit Recht die Stimmung unter den Beamten vor der Potsdamer Konferenz als „qualified pessimism" charakterisiert hat ${ }^{85}$, so war doch am Ende des Meinungswechsels mit den Stabschefs eine wichtige Vorentscheidung in der Frage gefallen, ob die britische Sicherheits- und Deutschlandpolitik nach dem Krieg vom Grundsatz der Sicherheit vor Deutschland oder eher vor der Sowjetunion geleitet sein müsse.

Die interne Erörterung dieser Frage im Foreign Office spielte zweitens die allgegenwärtige und historisch verankerte Sorge der Beamten um den Großmachtstatus des Inselreichs wider. Erwägungen der Machterhaltung waren es auch, die letztlich den Ausschlag zugunsten des Prinzips der Sicherheit vor Deutschland gaben und sie in einen Gegensatz zu den militärischen Planern treten ließen. Dabei wies der Streit mit den Chiefs of Staff zunächst auf einen fundamentalen Unterschied in der Ziel-Mittel-Relation beider Lager hin, der freilich bei genauerem Hinsehen einen strukturellen, von den Beteiligten jedoch nicht wahrgenommenen Widerspruch in beiden Positionen offenbarte. So verwarfen die Stabschefs jeden internationalistischen Lösungsweg. Sie sahen die geeignetsten Mittel, die sicherheitspolitischen Primärziele - Sicherung der Seeverbindungen und Schaffung ausreichender strategischer Tiefe in Kontinentaleuropa - zu erreichen, zum einen in einer Sicherheitspartnerschaft mit den USA, zum anderen in einer vorausschauenden Deutschlandpolitik, welche die schnellste militärische Nutzung der deutschen Kapazitäten gegen die Sowjetunion ermöglichte. Zugleich mochten sie von einer Frontstellung gegen die UdSSR die Bewahrung ihrer Einflußmöglichkeiten erhoffen. Dabei übersahen sie die innere Logik dieses Problemkomplexes, die einem gründlichen Beobachter eigentlich vor Augen führen mußte, daß gerade ein Kollisionskurs mit der Sowjetunion wirtschaftliche Belastungen nach sich ziehen mußte, die den Sog der wirtschaftlichen und strategischen Überlastung verstärken würden. Ein verteidigungspolitischer Alleingang mit Washington, in dem die Stabschefs einen konzeptionellen Fluchtpunkt für die Lösung der Sicherheitsfrage der Nachkriegszeit erblickten, ohne jedoch sichergehen zu können, ob der amerikanische Generalstab überhaupt mitzuziehen bereit war, mußte dann sein inneres Gleichgewicht verlieren und zu einem weiteren Beweis der machtpolitischen Deklassierung Großbritanniens umschlagen ${ }^{86}$. Es war also nicht nur die oft konstatierte Weitsicht der britischen Stabschefs, die sich hier zeigte, obwohl ihre Argumentation wichtige Aspekte der tatsächlich eintretenden Situation antizipierte; die Auseinandersetzung offenbarte in gleichem Maße eine von der Sorge um den künftigen Stand der Streitkräfte und die eigenen Einflußmöglichkeiten gespeiste Engstirnigkeit des militärischen Planungsansatzes. Von entscheidender Bedeutung sollte dabei sein, daß die militärische Führung an dieser Denkweise auch über das Kriegsende hinaus festhielt; aus

${ }^{85}$ Ross, S. 535.

${ }^{86}$ Für einen Hinweis, daß die Amerikaner dem Fortbestand der Combined Chiefs of Staff eher ablehnend gegenüberstanden, vgl. BM Add Mss 52578, Cunningham Papers, Diaries, 17.6.1945. 
den im letzten Kriegsjahr formulierten Sicherheitsplanungen entwickelte sich die strategische Gesamtplanung und vor allem die Bedarfsplanung für die Stärke der Streitkräfte ${ }^{87}$; sie trugen zur wachsenden Belastung der Zahlungsbilanz wie des Arbeitsmarktes bei.

Auf der anderen Seite hatte gerade die Befürchtung, daß eine Konfrontation mit den UdSSR kaum zumutbare ökonomische Folgen für das Königreich haben, den Wiederaufbauprozeß gefährden und zu ganz erheblichen machtpolitischen Einbußen führen mußte, die Mitarbeiter des Außenministeriums an der Vorstellung von einer britisch-sowjetischen Kooperation festhalten lassen. Dabei ging es ihnen nach innen um den Erhalt des außenpolitischen Primats, nach außen um die Rettung des britischen Großmachtstatus und die Kompensation entstandener Ressourcenlücken etwa durch die Schaffung eines westeuropäischen Regionalbündnisses, dem Großbritannien als Führungsmacht ohne sich freilich durch die Entsendung eines Expeditionsheeres in Friedenszeiten zu binden - gleichwohl vorstehen sollte. Die Widersprüche dieser Konzeption ließen sich jedoch kaum übersehen, denn eine Stabilisierungspolitik, wie sie das Außenministerium etwa mit dem Anspruch auf eine gemäßigte Reparationspolitik und der Ablehnung einer Aufteilung Deutschlands längst ins Auge gefaßt hatte, ließ sich auf Dauer mit dem Festhalten an einer britisch-sowjetischen Partnerschaft kaum vereinbaren. Dieser Widerspruch war vielleicht das hervorstechendste Element der britischen Außenpolitik am Ausgang des Krieges.

87 Vgl. dazu Ebersold, S. 141-186. 
